\title{
CYCLIC ACTIONS ON LENS SPACES
}

BY

PAIK KEE KIM

\begin{abstract}
A 3-dimensional lens space $L=L(p, q)$ is called symmetric if $q^{2} \equiv \pm 1 \bmod p$. Let $h$ be an orientation-preserving PL homeomorphism of even period $n(>2)$ on $L$ with nonempty fixed-point set. We show:

(1) If $n$ and $p$ are relatively prime, up to weak equivalence (PL), there exists exactly one such $h$ if $L$ is symmetric, and there exist exactly two such $h$ if $L$ is nonsymmetric.

(2) Fix $(h)$ is disconnected only if $p \equiv 0 \bmod n$, and there exists exactly one such $h$ up to weak equivalence (PL).

A $Z_{n}$-action is called nonfree if $\operatorname{Fix}(\phi) \neq \varnothing$ for some $\phi(\neq 1) \in Z_{n}$. We also classify all orientation-preserving nonfree $Z_{4}$-actions $(P L)$ on all lens spaces $L(p, q)$. It follows that each of $S^{3}$ and $P^{3}$ admits exactly three orientation-preserving $Z_{4}$-actions (PL), up to conjugation.
\end{abstract}

1. Introduction. All objects in this paper are in the PL category. Let $T$ be an orientation-preserving periodic homeomorphism of the 3-sphere $S^{3}$ with nonempty fixed-point set $\operatorname{Fix}(T)$. It is known that $\operatorname{Fix}(T)$ is a simple closed curve. A well-known conjecture, due to P. A. Smith, asserts that $\operatorname{Fix}(T)$ is unknotted (see Eilenberg [2]). It follows from a result of Waldhausen [23] that the conjecture is true for all homeomorphisms of even period on $S^{3}$.

Let $L=L(p, q)$ be a 3-dimensional lens space. Consider an orientationpreserving homeomorphism $h$ of period $n$ on $L(p, q)$ with $\operatorname{Fix}(h) \neq \varnothing$, where $p$ and $n$ are relatively prime. It can be seen that Fix $(h)$ is a simple closed curve and, if Smith's conjecture is true and $n>2$, then $\pi_{1}(L-\operatorname{Fix}(h))=Z$. In particular, $\pi_{1}(L-F i x(h))=Z$ is always true for all homeomorphisms $h$ of even period $(\neq 2)$ (see $\S 3$ ). In $\$ 3$, the following theorem will be proved:

THEOREM A. Let $h$ be an orientation-preserving homeomorphism of period $n$ on $L=L(p, q)$ with $(p, n)=1$, and $\pi_{1}(L-\operatorname{Fix}(h))=Z$. Then there exists exactly one such $h$ if $L$ is symmetric, and there exist exactly two such $h$ if $L$ is nonsymmetric, up to weak equivalence.

A lens space $L(p, q)$ is called symmetric if $q^{2} \equiv \pm 1 \bmod p$. Being symmetric is a topological property.

Let $k$ be a periodic homeomorphism of a space $X$. The cyclic group

Received by the editors July 6, 1974 and, in revised form, February 14, 1976 and July 5, 1976. AMS (MOS) subject classifications (1970). Primary 55C35, 57A10; Secondary 57E25.

Key words and phrases. Lens spaces, cyclic group action, periodic homeomorphism, fixed-point set.

- American Mathematical Society 1978 
generated by $k$ shall be denoted by $\langle k\rangle$. Two actions of $\langle k\rangle$ and $\left\langle k^{\prime}\right\rangle$ on $X$ are said to be conjugate if there exists a homeomorphism $t$ of $X$ such that $\left\langle t k t^{-1}\right\rangle=\left\langle k^{\prime}\right\rangle$. In this case, $k$ and $k^{\prime}$ are called weakly equivalent. If $t k t^{-1}=k^{\prime}$, then $k$ and $k^{\prime}$ are said to be equivalent.

Moise [13] proved that if $h: S^{3} \rightarrow S^{3}$ is periodic and preserves the orientation, and $\mathrm{Fix}(h)(\neq \varnothing)$ is unknotted, then $h$ is equivalent to a rotation (see P. A. Smith [19] for an alternative proof). This result is also obtained as a special type of Theorem A. The proof given here for this case seems shorter and more intuitive than the other two proofs.

In $\$ 3$ we also investigate all orientation-preserving homeomorphisms $h$ of period $n$ on $L=L(p, q)$ with $\pi_{1}(L-\operatorname{Fix}(h))=Z \oplus Z$. This is the case where $\operatorname{Fix}(h)$ is disconnected. In this case, $\pi_{1}(L-\operatorname{Fix}(h))=Z \oplus Z$ is always true for all homeomorphisms $h$ of even period $(\neq 2)$ (see §3).

THEOREM B. Let $h$ be an orientation-preserving homeomorphism of period $n$ on $L=L(p, q)$, and $\pi_{1}(L-F i x(h))=Z \oplus Z$. Then there exists such an $h$ if and only if $p \equiv 0 \bmod n$, and $h$ is unique up to weak equivalence.

A $Z_{n}$-action on $L$ shall be called nonfree if $\operatorname{Fix}(\phi) \neq \varnothing$ for some $\phi$ $(\neq 1) \in Z_{n}$. In $\S 4$, we classify all orientation-preserving nonfree $Z_{4}$-actions on all lens spaces $L(p, q)$. We summarize the result of $\$ 4$ as the following:

THEOREM C. The number of nonconjugate, orientation-preserving, nonfree $Z_{4}$-actions on $L=L(p, q)$ is given as the following:

(1) For $p$ odd, there are two if $L$ is symmetric and four if $L$ is nonsymmetric.

(2) For $p=2 s$ ( $s$ odd), there is one if $L$ is symmetric and two if $L$ is nonsymmetric.

(3) For $p \equiv 0 \bmod 4$, there are three if $q \equiv a d+b c \bmod p$ where $p=2 a c$ and $a d-b c=1$, and there are four if $q \equiv a d+b c-(a b / 2) \bmod p$ where $p=2 a c(a$ even) and ad $-b(c+a / 2)=1$ (the homeomorphic type of $L(p, q)$ does not depend on the choice of $b, d)$.

Otherwise, there exist only two nonfree $Z_{4}$-actions.

All $Z_{2}$-actions on the 3-sphere $S^{3}$ [9], [10], [22] and the projective 3-space $P^{3}$ [5], [7] are known. As results of $\S 4$, we have the following (see Rice [17] for free $Z_{4}$-actions on $S^{3}$ ).

THEOREM D. Each of $S^{3}$ and $P^{3}$ admits exactly three orientation-preserving $Z_{4}$-actions, up to conjugation.

A homeomorphism $h$ of $L=L(p, q)$ is called sense-preserving if $h$ induces the identity on $H_{1}(L)$.

Let $h$ be a periodic (PL) homeomorphism of a finite simplicial complex $M$. It can be shown that $h$ becomes simplicial after a suitable subdivision $M_{0}$ 
such that $h$ is regular, i.e., $h(\sigma)=\sigma$ for a simplex $\sigma \in M_{0}$ only if $h \mid \sigma$ is the identity.

I would like to thank Professors K. W. Kwun and J. L. Tollefson for conversations.

2. $Z_{n}$-actions on $D^{2} \times S^{1}$. Let $D^{2}$ be the unit disk in the Gaussian plane of complex numbers and $S^{1}$ its boundary. $D^{2} \times S^{1}$ is the solid torus whose points can be denoted by $\left(\rho z_{1}, z_{2}\right)$ where $z_{1}, z_{2} \in S^{1}$ and $0<\rho<1$.

Let $h$ be an orientation-preserving homeomorphism of period $n$ on a compact $n$-manifold $X$ with Fix $(h) \neq \varnothing$. Let $q$ be a prime factor of $n$ such that $n=m q$. Then $h^{m}$ is of prime period $q$. It is known that $\Sigma_{k} \operatorname{dim} H_{k}(F)<$ $\Sigma_{k} \operatorname{dim} H_{k}(X)$ over $Z_{q}$ where $F=\operatorname{Fix}\left(h^{m}\right)$ (for the proof, see [1]). Now, let $X=D^{2} \times S^{1}$, and assume that $\operatorname{Fix}(h) \subset \operatorname{Int}\left(D^{2} \times S^{1}\right)$. It is easy to see that $\operatorname{Fix}(h)$ is a 1 -manifold. Since $\operatorname{Fix}(h) \subset \operatorname{Fix}\left(h^{m}\right)$ and the above inequality holds, Fix $(h)$ is a simple closed curve, and Fix $(h)=\operatorname{Fix}\left(h^{i}\right), 0<i<n$.

DEFINITION 2.1. Let $h$ be a homeomorphism of period $n$ on a 3-manifold $M$. Let $g: M \rightarrow M /\left\langle h^{m}\right\rangle$ be the orbit map induced by $\left\langle h^{m}\right\rangle$. Then there exists a homeomorphism $\bar{h}$ of $M /\left\langle h^{m}\right\rangle$, uniquely determined by $h$, such that $\bar{h} g=g h$. The map $\bar{h}$ shall be called the homeomorphism on $M /\left\langle h^{m}\right\rangle$ induced by $h$.

Let $h$ be an orientation-preserving homeomorphism of period $n$ on $D^{2} \times$ $S^{1}$ with $F=\operatorname{Fix}(h) \neq \varnothing$. Suppose that $\left(D^{2} \times S^{1}, F\right) \approx\left(D^{2} \times S^{1}, 0 \times S^{1}\right)$ (i.e., they are homeomorphic as a pair). We may assume that $h$ is regular after a suitable subdivision. Let $U$ be a simplicial (regular) neighborhood of $F$. Let $M=D^{2} \times S^{1} /\langle h\rangle$ and $g: D^{2} \times S^{1} \rightarrow M$ be the orbit map. Since $M$ is an orientable manifold and $g(U)$ is a simplicial neighborhood of $g(F), g(U)$ is a solid torus. We have the following two lemmas.

Lemma 2.2. The orbit space $M=D^{2} \times S^{1} /\langle h\rangle$ is a solid torus.

Proof. Let $U^{\prime}=g(U)$. We claim that $\pi_{1}\left(M-U^{\prime}\right)=Z \oplus Z$. Since $M-$ $U^{\prime}$ has two boundary components, $H_{2}\left(M-U^{\prime}\right)$ is of rank $>1$. Since $\chi\left(M-U^{\prime}\right)=0, H_{1}\left(M-U^{\prime}\right)$ is of rank $>2$. Therefore, since we have an obvious short exact sequence

$$
0 \rightarrow Z \oplus Z \rightarrow \pi_{1}\left(M-U^{\prime}\right) \rightarrow Z_{n} \rightarrow 0,
$$

$\pi_{1}\left(M-U^{\prime}\right)$ is an abelian group of rank 2 . As $M-U^{\prime}$ is covered by a contractible space and no nontrivial finite group can act freely on a finitedimensional, contractible space (due to P. A. Smith [4]), $\pi_{1}\left(M-U^{\prime}\right)$ has no torsion subgroup, and $\pi_{1}\left(M-U^{\prime}\right)=Z \oplus Z$. Therefore, since $\mathrm{cl}\left(M-U^{\prime}\right)$ is irreducible, $\operatorname{cl}\left(M-U^{\prime}\right)$ may be obtained from $S^{1} \times I_{1} \times I_{2}$ by identifying each $(x, 0)\left(x \in S^{1} \times I_{1}\right)$ with $(f(x), 1)$, where $I_{i}(i=1,2)$ is the unit interval $I$ and $f$ is a homeomorphism of $S^{1} \times I_{1}$ (see Stallings [20]). Since $\operatorname{cl}\left(M-U^{\prime}\right)$ has two boundary components, $f$ carries $S^{1} \times\{i\}$ onto $S^{1} \times\{i\}$ 
$(i=0,1)$. Since $\operatorname{cl}\left(M-U^{\prime}\right)$ is orientable, $f$ must preserve the orientation, and it can be shown that $f$ is isotopic to the identity. Therefore, $\operatorname{cl}\left(M-U^{\prime}\right)$ $\approx S^{1} \times S^{1} \times I$, and $M$ is a solid torus.

LEMma 2.3. There exists a disk $E$ properly embedded in $D^{2} \times S^{1}$ such that $h(E)=E$ and $\partial E$ does not bound a disk in $S^{1} \times S^{1}$.

One may easily derive the proof of the above lemma from that of Lemma 2.8 , and we omit the proof.

Proposition 2.4. Let $h$ be an orientation-preserving homeomorphism of period $n$ on $D^{2} \times S^{1}$ with $F=\operatorname{Fix}(h) \neq \varnothing$. Suppose that

$$
\left(D^{2} \times S^{1}, F\right) \approx\left(D^{2} \times S^{1}, 0 \times S^{1}\right) .
$$

Then there exists a unique such $h$, up to weak equivalence.

Proof. We may assume that $F=0 \times S^{1}$. Let $h_{i}(i=1,2)$ be two homeomorphisms such as $h$. By Lemma 2.2, each orbit space

$$
M_{i}=D^{2} \times S^{1} /\left\langle h_{i}\right\rangle
$$

is a solid torus. Parametrize $M_{i}$ in terms of $D^{2} \times S^{1}$. Let $(1,0)$ and $(0,1)$ be the canonical generators of $\pi_{1}\left(S^{1} \times S^{1}\right)$ (we disregard the base point as $\pi_{1}\left(S^{1} \times S^{1}\right)$ is abelian). Let $g_{i}^{\prime}: D^{2} \times S^{1} \rightarrow M_{i}=D^{2} \times S^{1}$ be the orbit map and $g_{i}=g_{i}^{\prime}\left(D^{2}-0\right) \times S^{1}$. By Lemma 2.3 , we may assume that $g_{i \#}[(1,0)]=$ $(n, 0)$. Let $g_{i \#}[(0,1)]=\left(a_{i}, b_{i}\right)$. Since $g_{i}$ is a covering projection with $n$ sheets, $(1,0)$ and $\left(a_{i}, b_{i}\right)$ generate $\pi_{1}\left(S^{1} \times S^{1}\right)$, and therefore we may assume that $b_{i}=1$. Define $t^{\prime}$ of $M_{1}$ to $M_{2}$ by

$$
t^{\prime}\left(\rho z_{1}, z_{2}\right)=\left(\rho z_{1} z_{2}^{a_{2}-a_{1}}, z_{2}\right) \text { for }\left(\rho z_{1}, z_{2}\right) \in D^{2} \times S^{1} .
$$

Since $\left(t g_{1}\right)_{\#}=g_{2 \#}$, where $t=t^{\prime} \mid\left(D^{2}-0\right) \times S^{1}$, it follows from the lifting theorem that there exists a homeomorphism $\bar{t}$ of $\left(D^{2}-0\right) \times S^{1}$ such that $g_{2} \bar{t}=t g_{1}$. Therefore, $\bar{t} h_{1} \bar{t}^{-1}=\alpha\left(h_{2}\right)$ for some automorphism $\alpha$ of $\left\langle h_{2}\right\rangle$. One may extend $\bar{t}$ to $D^{2} \times S^{1}$ such that $\overline{t h}_{1} \bar{t}^{-1}=\alpha\left(h_{2}\right)$ on $D^{2} \times S^{1}$. This completes the proof.

(2.5). Let $h$ be an involution on $D^{2} \times S^{1}$. It follows from a result of [6] that $h$ is equivalent to an involution of a form $h_{1} \times h_{2}$ on $D^{2} \times S^{1}$ (see also [9], [10], [22]). Especially $D^{2} \times S^{1}$ admits exactly two (nonconjugate) obvious orientation-preserving involutions $h$ with $\operatorname{Fix}(h) \neq \varnothing$ (see also [21]).

COROLlaRY 2.6. There exists exactly one orientation-preserving homeomorphism $h$ of period $2 k(k \neq 1)$ on $D^{2} \times S^{1}$ with $\operatorname{Fix}(h) \neq \varnothing$, up to weak equivalence.

Proof. If $\operatorname{Fix}\left(h^{k}\right) \subset \operatorname{Int}\left(D^{2} \times S^{1}\right)$, we see that $\operatorname{Fix}(h)=\operatorname{Fix}\left(h^{k}\right)$, and the result follows from (2.5) and Proposition 2.4. Indeed, we will point out that $\operatorname{Fix}(h) \subset \operatorname{Int}\left(D^{2} \times S^{1}\right)$. Suppose that $\operatorname{Fix}(h) \cap S^{1} \times S^{1} \neq \varnothing$. Then $\operatorname{Fix}\left(h^{k}\right)$ 
is a disjoint union of two arcs and $D^{2} \times S^{1} /\left\langle h^{k}\right\rangle$ is homeomorphic to $D^{3}$ (see (2.5)). Since $h$ is orientation-preserving and $\operatorname{Fix}(h) \subset \operatorname{Fix}\left(h^{k}\right)$, we see that $\operatorname{Fix}(h)=\operatorname{Fix}\left(h^{k}\right)$. Let $\bar{h}$ be the homeomorphism on $D^{2} \times S^{1} /\left\langle h^{k}\right\rangle$ induced by $h$ and $g: D^{2} \times S^{1} \rightarrow D^{2} \times S^{1} /\left\langle h^{k}\right\rangle$ be the orbit map. Since $g(\operatorname{Fix}(h)) \subset$ Fix $(\bar{h})$, Fix $(\bar{h})$ has at least two components. This is a contradiction since $\bar{h}$ is a periodic map of $D^{2} \times S^{1} /\left\langle h^{k}\right\rangle \approx D^{3}$ (Fix $(\bar{h})$ must be an arc in this case).

Since the proof of the following lemma is easy, we omit it.

LEMMA 2.7. Let $h$ be a homeomorphism of period $n$ on $D^{2} \times S^{1}$. If $\langle h\rangle$ acts freely on $D^{2} \times S^{1}$, then $h$ is given by $h\left(\rho z_{1}, z_{2}\right)=\left(\rho z_{1}, \omega z_{2}\right)$ or $h\left(\rho z_{1}, z_{2}\right)=$ $\left(\rho \bar{z}_{1}, \omega z_{2}\right)$ for $\left(\rho z_{1}, z_{2}\right) \in D^{2} \times S^{1}$, where $\omega=e^{2 \pi i / n}$, up to weak equivalence.

Let $h$ be an orientation-preserving homeomorphism of period $2^{n}(n \neq 1)$ on $D^{2} \times S^{1}$. Let $G=\left\{h^{i} \mid \mathrm{Fix}\left(h^{i}\right) \neq \varnothing\right\}$. Since $G$ forms a group, $G=\left\langle h^{2^{k}}\right\rangle$ for some $k, 0<k \leqslant n$. One can see that $\operatorname{Fix}\left(h^{2^{k}}\right) \subset \operatorname{Int}\left(D^{2} \times S^{1}\right.$ ) (use a similar argument to that in the proof of Corollary 2.6; the induced homeomorphism $\bar{h}$ on $D^{2} \times S^{1} /\left\langle h^{2^{n-1}}\right\rangle$ may be free this time). Therefore, it follows from (2.5) and Corollary 2.6 that $\left(D^{2} \times S^{1}\right.$, Fix $\left.\left(h^{2^{k}}\right)\right) \approx\left(D^{2} \times S^{1}, 0 \times S^{1}\right)$. We may assume that $h$ is regular after a suitable subdivision of $D^{2} \times S^{1}$. Let $\bar{h}$ be the homeomorphism on $M_{1}=D^{2} \times S^{1} /\left\langle h^{2^{k}}\right\rangle$ induced by $h$. By Lemma $2.2, M_{1}$ is a solid torus. Note that $\bar{h}$ is an orientation-preserving homeomorphism of period $2^{k}$. Since $\langle\bar{h}\rangle$ acts freely on $M_{1}$, it follows from Lemma 2.7 that $M_{2}=M_{1} /\langle\bar{h}\rangle$ is a solid torus. Let $g_{1}: D^{2} \times S^{1} \rightarrow M_{1}$ and $g_{2}: M_{1} \rightarrow M_{2}$ be the orbit maps. Then the composite map $g=g_{2} g_{1}$ is the orbit map of $D^{2} \times S^{1} \rightarrow D^{2} \times S^{1} /\langle h\rangle$. Let $F=\operatorname{Fix}\left(h^{2^{k}}\right)$. Since $F$ is invariant under $h$, $g_{1}(F)$ is invariant under $h$. We have the following lemma.

LEMMA 2.8. There exists a disk $E$ properly embedded in $D^{2} \times S^{1}$ such that $h^{i}(E) \cap E=\varnothing, 1 \leqslant i<2^{k}$, and $h^{2^{k}}(E)=E$, and $\partial E$ does not bound a disk in $S^{1} \times S^{1}$.

Proof. It is easy to find a disk $E^{\prime}$ properly embedded in a simplicial neighborhood $U$ of $F$ after a suitable subdivision of $D^{2} \times S^{1}$ such that $h^{i}\left(E^{\prime}\right) \cap E^{\prime}=\varnothing, 1 \leqslant i<2^{k}$, and $h^{2^{k}}\left(E^{\prime}\right)=E^{\prime}$, and $\partial E^{\prime}$ does not bound a disk in $\partial U$. Then $g\left(E^{\prime}\right)$ is a disk properly embedded in $g(U)$. Since $\operatorname{cl}\left(M_{2}-\right.$ $g(U)$ ) is a boundary collar of $g(U)$ (see the proof of Lemma 2.2), there exists a disk $E_{2}$ properly embedded in $M_{2}$ such that $g\left(E^{\prime}\right) \subset \operatorname{Int}\left(E_{2}\right)$ and $\partial E_{2}$ does not bound a disk in $\partial M_{2}$. Since $g_{2}$ is a covering projection with $2^{k}$ sheets, $g_{2}^{-1}\left(E_{2}\right)$ is $2^{k}$ disjoint copies of disks properly embedded in $M_{1}$. Let $E_{1}$ be the disk which is a component of $g_{2}^{-1}\left(E_{2}\right)$ and $E_{1} \cap g_{1}\left(E^{\prime}\right) \neq \varnothing$. Let $E=$ $g_{1}^{-1}\left(E_{1}\right)$. Since $g_{1} \mid\left(E^{\prime}-F\right)$ is a covering projection, $E$ must be connected, and it is a disk as desired. 
THEOREM 2.9. There exist exactly three orientation-preserving $Z_{4}$-actions on $D^{2} \times S^{1}$, up to conjugation.

Proof. By Corollary 2.6 and Lemma 2.7, it is enough to show that two orientation-preserving homeomorphisms $h_{i}(i=1,2)$ of period 4 on $D^{2} \times S^{1}$, which satisfy $\operatorname{Fix}\left(h_{i}\right)=\varnothing$ and $\operatorname{Fix}\left(h_{i}^{2}\right) \neq \varnothing$, are weakly equivalent. Since $\operatorname{Fix}\left(h_{i}^{2}\right) \subset \operatorname{Int}\left(D^{2} \times S^{1}\right)$, we may assume that

$$
\operatorname{Fix}\left(h_{i}^{2}\right)=0 \times S^{1} \subset D^{2} \times S^{1} \text { for each } i
$$

(see (2.5)). Let $\bar{M}_{i}=D^{2} \times S^{1} /\left\langle h_{i}^{2}\right\rangle$ and $\bar{h}_{i}$ be the homeomorphism on $\bar{M}_{i}$ induced by $h_{i}$. Let $M_{i}=\bar{M}_{i} /\left\langle\overline{h_{i}}\right\rangle$. Let $\bar{g}_{i}: D^{2} \times S^{1} \rightarrow \bar{M}_{i}$ and $g_{i}: \bar{M}_{i} \rightarrow M_{i}$ be the orbit maps, and $f_{i}=g_{i} \bar{g}_{i}$. Parametrize $\bar{M}_{i}$ and $M_{i}$ in terms of $D^{2} \times S^{1}$. Let $(1,0)$ and $(0,1)$ be the canonical generators of $\pi_{1}\left(S^{1} \times S^{1}\right)$. Let

$$
\bar{g}_{i}^{\prime}=\bar{g}_{i}\left|\left(D^{2}-0\right) \times S^{1}, g_{i}^{\prime}=g_{i}\right|\left(D^{2}-0\right) \times S^{1} \text {, and } f_{i}^{\prime}=f_{i} \mid\left(D^{2}-0\right) \times S^{1} \text {. }
$$

By Lemma 2.8, we assume that $f_{i \#}^{\prime}[(1,0)]=(2,0)$. Let $f_{i \#}^{\prime}[(0,1)]=\left(a_{i}, b_{i}\right)$. Since the index of $f_{i \#}^{\prime}\left(\pi_{1}\left(S^{1} \times S^{1}\right)\right)$ in $\pi_{1}\left(\partial M_{i}\right)$ is 4 , we may assume that $b_{i}=2$. Since $f_{i \#}^{\prime}[(1,0)]=(2,0)$ and $f_{i \#}^{\prime}[(0,1)]=\left(a_{i}, 2\right)$, each $a_{i}$ must be odd (otherwise, the covering transformation group of the covering space $\left(\left(D^{2}-\right.\right.$ $\left.0) \times S^{1}, f_{i}^{\prime}\right)$ would be $Z_{2} \oplus Z_{2}$ ). Take base points $\hat{x}_{i} \in S^{1} \times S^{1} \subset D^{2} \times$ $S^{1}, \bar{x}_{i} \in \partial \bar{M}_{i}$ and $x_{i} \in \partial M_{i}$ such that $\bar{g}_{i}\left(\hat{x}_{i}\right)=\bar{x}_{i}$ and $g_{i}\left(\bar{x}_{i}\right)=x_{i}$. Define a homeomorphism $t$ of $M_{1}$ to $M_{2}$ such that

$$
t\left(\rho z_{1}, z_{2}\right)=\left(\rho z_{1} z_{2}^{\left(a_{2}-a_{1}\right) / 2}, z_{2}\right) \text {. }
$$

Then, by letting $t^{\prime}=t \mid\left(M_{1}-0 \times S^{1}\right)$, we see that $t_{\#}^{\prime} f_{1 \#}^{\prime}=f_{2 \#}^{\prime}$, and therefore there exists a lifting homeomorphism $\hat{t}$ of $\left(D^{2}-0\right) \times S^{1}$ such that $f_{2}^{\prime} \hat{t}=t^{\prime} f_{1}^{\prime}$ and $\hat{t}\left(\hat{x}_{1}\right)=\hat{x}_{2}$. On the other hand, since each $g_{i}$ is a covering projection, there exists a lifting homeomorphism $\bar{t}$ of $\bar{M}_{1}$ to $\bar{M}_{2}$ such that $t_{1}=g_{2} \bar{t}$ and $\bar{t}\left(\bar{x}_{1}\right)=\bar{x}_{2}$. We may assume that $\bar{t}\left(0 \times S^{1}\right)=0 \times S^{1}$. Since $f_{2} \hat{t}=t f_{1}$ on $\left(D^{2}-0\right) \times S^{1}, \hat{t h} \hat{t}^{-1}=h_{2}^{j}$ on $\left(D^{2}-0\right) \times S^{1}, j=1$ or 3 . Therefore, there exists an obvious homeomorphism $t_{0}$ of $\bar{M}_{1}-0 \times S^{1}$ to $\bar{M}_{2}-0 \times S^{1}$ induced by $\hat{t}$ such that $\bar{g}_{2} \hat{t}=t_{0} \bar{g}_{1}$ on $\left(D^{2}-0\right) \times S^{1}$ and $g_{2} t_{0}=\operatorname{tg}_{1}$ on $\left(D^{2}-0\right) \times S^{1}$. Since $\hat{t}\left(\hat{x}_{1}\right)=\hat{x}_{2}$, and $t_{0}\left(\bar{x}_{1}\right)=\bar{x}_{2}$, it follows from the unique lifting property that $t_{0}=\bar{t} \mid\left(D^{2}-0\right) \times S^{1}$. Since $\bar{t}\left(0 \times S^{1}\right)$ $=0 \times S^{1}$, one may easily extend $\hat{t}$ to a homeomorphism $\tilde{t}$ of $D^{2} \times S^{1}$ such that the following diagram commutes:

$$
\begin{array}{ccccc}
\left(D^{2} \times S^{1}, \hat{x}_{1}\right) & \stackrel{\bar{g}_{1}}{\rightarrow} & \left(\bar{M}_{1}, \bar{x}_{1}\right) & \stackrel{g_{1}}{\rightarrow} & \left(M_{1}, x_{1}\right) \\
\downarrow \tilde{t} & & \downarrow \bar{t} & & \downarrow t \\
\left(D^{2} \times S^{1}, \hat{x}_{2}\right) & \stackrel{\bar{g}_{2}}{\rightarrow} & \left(\bar{M}_{2}, \bar{x}_{2}\right) & \stackrel{g_{2}}{\rightarrow} & \left(M_{2}, x_{2}\right)
\end{array}
$$

Hence $\tilde{t}_{1} \tilde{t}^{-1}=h_{2}^{j}, j=1$ or 3 . This completes the proof. 
3. $Z_{n}$-actions on $L(p, q)$. Let $h$ be an orientation-preserving homeomorphism of period $n$ on $L=L(p, q)$ with $\operatorname{Fix}(h) \neq \varnothing$. Then $\operatorname{Fix}(h)$ is a disjoint union of at most two simple closed curves (cf. the proof of Proposition 3.1).

Proposition 3.1. If $(n, p)=1$, then $\operatorname{Fix}\left(h^{m}\right)(0<m<n)$ is a simple closed curve.

Proof. Let $T=h^{m}, 0<m<n$. Let $r$ be the prime period ( $\left.>1\right)$ of $T^{k}$ for some $k$. Let $F=\operatorname{Fix}\left(T^{k}\right)$. Since $\Sigma_{i} \operatorname{dim} H_{i}(F) \leqslant \Sigma_{i} \operatorname{dim} H_{i}(L)$ over $Z_{r}$ and $(r, p)=1, F$ is a simple closed curve and so is $\operatorname{Fix}(T)$.

Proposition 3.2. If $n$ is even $(>2)$, then $\pi_{1}(L-F i x(h))=Z$ or $Z \oplus Z$.

Proof. See (3.3).

(3.3). Let $n>2$. Let $h_{\#}(a)=k a$ for $a \in \pi_{1}(L)$. It follows from a result of Olum [14, p. 467] that $k^{2} \equiv \operatorname{deg} h \bmod p$, and $h^{2}$ is sense-preserving. Let $F_{0}$ be a component of $\operatorname{Fix}(h)$. By the lifting theorem, there exists a lifting $T$ : $\left(S^{3}, y\right) \rightarrow\left(S^{3}, y\right)$ of period $n$ with $\operatorname{Fix}(T) \neq \varnothing$ such that $g T=h g$, where $g$ : $S^{3} \rightarrow L$ is the natural projection and $g(y) \in F_{0}$. Since $F_{0} \subset \operatorname{Fix}\left(h^{2}\right)$ and $h^{2}$ is sense-preserving, we see that $g^{-1}\left(F_{0}\right)$ is connected (for a proof, see [8]). Let $\tilde{F}=g^{-1}\left(F_{0}\right)$. Then $\tilde{F}$ is the fixed-point set of $T$. Assume that Smith's conjecture is true (this is true if $n$ is even). Then $\pi_{1}\left(S^{3}-\tilde{F}\right)=Z$ (see [15D). Since there is an obvious short exact sequence

$$
0 \rightarrow Z \rightarrow \pi_{1}\left(L-F_{0}\right) \rightarrow Z_{p} \rightarrow 0,
$$

we see that $\pi_{1}\left(L-F_{0}\right)$ is an abelian group of rank 1 . Since $L-F_{0}$ is covered by a contractible space, $\pi_{1}\left(L-F_{0}\right)$ has no nontrivial element of finite order (see [4, p. 287]), and $\pi_{1}\left(L-F_{0}\right)=Z$. Let $U$ be a regular (invariant) neighborhood of $F_{0}$ in $L$ such that $U \cap \operatorname{Fix}(h)=F_{0}$. Since $L-F_{0}$ is irreducible, it follows from a result of Stallings [20] that $\operatorname{cl}(L-U)$ is a solid torus. Therefore, if $\operatorname{Fix}(h)$ is not connected and $n$ is even, it follows from (2.5) that $\pi_{1}(L-\operatorname{Fix}(h))=Z \oplus Z$.

Proposition 3.2 is not true for involutions. It is known that an involution $h$ is sense-preserving if and only if $\pi_{1}(L-F i x(h))=Z$ or $Z \oplus Z$ (see [5], [8]). Every lens space $L(p, q)(p>2)$ admits an orientation-preserving involution which is not sense-preserving and has nonempty fixed-point set.

The proofs of Theorem A and Theorem B will be given in the following two parts I, II.

I. Proof of Theorem A. Let $F=\operatorname{Fix}(h)$. By Proposition $3.1, F$ is a simple closed curve. Let $g: S^{3} \rightarrow L$ be the natural projection. Since $\pi_{1}(L-F)=Z$, we see that $\pi_{1}\left(S^{3}-g^{-1}(F)\right)=Z$ and $g^{-1}(F)$ is connected. Therefore, $S^{3}-$ $g^{-1}(F)$ is irreducible, and so is $L-F$. Let $U$ be an invariant regular neighborhood of $F$ in $L$. Since $\operatorname{cl}(L-U)$ is irreducible and $\pi_{1}(L-U)=Z$, 
it follows that $\operatorname{cl}(L-U)$ is a solid torus (see [20]). Hence $L$ may be given as $L=D^{2} \times S^{1} \cup_{k} S^{1} \times D^{2}$ for an attaching map $k$ of $S^{1} \times S^{1}$, where $D^{2} \times$ $S^{1}$ is an invariant regular neighborhood of $F$. Let $h_{1}=h \mid D^{2} \times S^{1}$ and $h_{2}=h \mid S^{1} \times D^{2}$. By Proposition 2.4 and Lemma 2.7, we may assume that

$$
L=D^{2} \times S^{1} \cup S^{1} \times D^{2}
$$

for an appropriate attaching map $f$ of $S^{1} \times S^{1}$ and $h$ is given by

$$
\begin{array}{ll}
e\left(h_{1}\right)\left(\rho z_{1}, z_{2}\right)=\left(\rho \omega z_{1}, z_{2}\right) & \text { on } D^{2} \times S^{1} \text { and } \\
e^{\prime}\left(h_{2}\right)\left(z_{1}, \rho z_{2}\right)=\left(\omega z_{1}, \rho z_{2}\right) & \text { on } S^{1} \times D^{2}
\end{array}
$$

where $\omega=e^{2 \pi i / n}$, and $e, e^{\prime}$ are some automorphisms of $\left\langle h_{1}\right\rangle$ and $\left\langle h_{2}\right\rangle$, respectively.

Let $(1,0)$ and $(0,1)$ be the elements of $\pi_{1}\left(S \times S^{1}\right)=Z \oplus Z$, represented by the paths $\left(e^{2 \pi i t}, 1\right)$ and $\left(1, e^{2 \pi i t}\right)(0<t<1)$, respectively. Suppose that $f_{\#}[(1,0)]=(a, b)$ and $f_{\#}[(0,1)]=(c, d) . f_{\#}$ may be written as

$$
f_{\#}=\left(\begin{array}{ll}
a & c \\
b & d
\end{array}\right)
$$

Then

$$
\left|\begin{array}{ll}
a & c \\
b & d
\end{array}\right|= \pm 1 \text {. }
$$

Let $g_{1}: D^{2} \times S^{1} \rightarrow D^{2} \times S^{1} /\left\langle h_{1}\right\rangle$ and $g_{2}: S^{1} \times D^{2} \rightarrow S^{1} \times D^{2} /\left\langle h_{2}\right\rangle$ be the orbit maps. Let $g_{i}^{\prime}=g_{i} \mid S^{1} \times S^{1}(i=1,2)$. Then there are obvious parametrizations of $D^{2} \times S^{1} /\left\langle h_{1}\right\rangle$ and $S^{1} \times D^{2} /\left\langle h_{2}\right\rangle$ in terms of $D^{2} \times S^{1}$ and $S^{1} \times D^{2}$, respectively, such that $g_{i \#}^{\prime}[(1,0)]=(n, 0)$ and $g_{i \#}^{\prime}[(0,1)]=(0,1)$. We have:

LEMMA 3.4. $b \equiv 0 \bmod n$.

Proof. Consider the following commutative diagram:

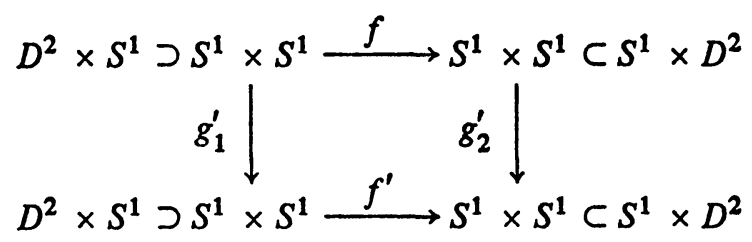

where $f^{\prime}$ is the induced attaching map of $S^{1} \times S^{1}$ in $L /\langle h\rangle$.Chasing the above diagram, it can be shown that $a=a^{\prime}, b=n b^{\prime}, n c=c^{\prime}$ and $d=d^{\prime}$, where

$$
f_{\#}^{\prime}=\left(\begin{array}{ll}
a^{\prime} & c^{\prime} \\
b^{\prime} & d^{\prime}
\end{array}\right)
$$

(3.5). Let $X=D^{2} \times S^{1} \cup_{k} S^{1} \times D^{2}$ where 


$$
k_{\#}=\left(\begin{array}{ll}
\alpha & \gamma \\
\beta & \delta
\end{array}\right) .
$$

By Mangler [11], the isotopy classes of homeomorphisms of $S^{1} \times S^{1}$ are precisely the automorphism classes of $\pi_{1}\left(S^{1} \times S^{1}\right)$. Therefore, the integers $\alpha, \beta, \gamma$, and $\delta$ completely determine the isotopy class of $k$. It can be shown that $X \approx L(\alpha, \beta)$ if $\alpha \neq 0$. We denote $X$ by $L(\alpha, \gamma, \beta, \delta)$ if $k$ is given by

$$
k\left(z_{1}, z_{2}\right)=\left(z_{1}^{\alpha} z_{2}^{\gamma}, z_{1}^{\beta} z_{2}^{\delta}\right) \text { for }\left(z_{1}, z_{2}\right) \in S^{1} \times S^{1} \text {. }
$$

Recall that $L(\alpha, \beta)$ is homeomorphic to $L\left(\alpha, \beta^{\prime}\right)$ if and only if $\beta \equiv \pm \beta^{\prime}$ or $\beta \beta^{\prime} \equiv \pm 1 \bmod \alpha$ [12], [16].

Define a homeomorphism $h_{0}$ on $L_{0}=L(a, c, b, d)$ by

$$
\begin{array}{ll}
h_{0}\left(\rho z_{1}, z_{2}\right)=\left(\rho \omega z_{1}, z_{2}\right) & \text { on } D^{2} \times S^{1} \text { and } \\
h_{0}\left(z_{1}, \rho z_{2}\right)=\left(\omega^{a} z_{1}, \rho z_{2}\right) & \text { on } S^{1} \times D^{2}
\end{array}
$$

where $\omega=e^{2 \pi i / n}$. By Lemma 3.4, $h_{0}$ is well-defined. Since $a= \pm p$ (see (3.5)), and $n, a$ are relatively prime, the period of $h_{0}$ is $n$. We shall denote $h_{0}$ by $h(a, c, b, d)$.

LEMMA 3.6. $h$ is weakly equivalent to $h_{0}=h(a, c, b, d)$.

Proof. The orbit spaces $L /\langle h\rangle$ and $L_{0} /\left\langle h_{0}\right\rangle$ are given as $D^{2} \times S^{1} U_{f^{\prime}} S^{1}$ $\times D^{2}$ and $D^{2} \times S^{1} \cup_{f_{0}^{\prime}} S^{1} \times D^{2}$, respectively. By the proof of Lemma 3.4, $f^{\prime}$ and $f_{0}^{\prime}$ are isotopic. Therefore, there exists a homeomorphism $t^{\prime}: L /\langle h\rangle \rightarrow$ $L_{0} /\left\langle h_{0}\right\rangle$ such that $t^{\prime}\left(D^{2} \times S^{1}\right)=D^{2} \times S^{1}$ and $t^{\prime}\left(0 \times S^{1}\right)=0 \times S^{1}$. Since $S^{1} \times D^{2}$ is a deformation retract of $L-F i x(h)$ (and $L_{0}-\operatorname{Fix}\left(h_{0}\right)$ ), one may obtain a lifting homeomorphism $t$ of $L$ to $L_{0}$ (by the lifting theorem) such that $t h t^{-1}=e\left(h_{0}\right)$ where $e$ is an automorphism of $\left\langle h_{0}\right\rangle$.

(3.7). We observe that there exists such a map $h$ of period $n$ on $L=L(p, q)$ ( $p$ and $n$ are relatively prime). Since $(p, n q)=1$, there exist integers $r, s$ such that $p r-(n s) q=1$. Consider the homeomorphism $\tilde{h}=h(p, q, n s, r)$ of $L(p, q, n s, r)$. Since $L(p, q, n s, r) \approx L(p, n s)$ and $L(p, n s) \approx L(p, q)$, there exists a homeomorphism $t$ of $L$ to $L(p, q, n s, r)$. Then the homeomorphism $t^{-1} \tilde{h} t$ is a homeomorphism as desired.

By Lemma 3.6, our classification problem now depends on classifying the homeomorphisms $h(a, c, b, d)$ for various possible integers $a, b, c$ and $d$, where $a d-b c= \pm 1$. Our information on the integers is that $a= \pm p$ and $b \equiv 0 \bmod n$. If $h(a, c, b, d)$ is weakly equivalent to $h\left(a^{\prime}, c^{\prime}, b^{\prime}, d^{\prime}\right)$, we shall denote the fact by $h(a, c, b, d) \sim h\left(a^{\prime}, c^{\prime}, b^{\prime}, d^{\prime}\right)$.

LEMMA 3.8. (1) $h(a, c, b, d) \sim h(-a, c,-b, d)$.

(2) $h(a, c, b, d) \sim h(a,-c, b,-d)$.

Proof. For (1), define $t$ of $L(a, c, b, d)$ to $L(-a, c,-b, d)$ by $t\left(\rho z_{1}, z_{2}\right)=$ 
$\left(\rho z_{1}^{-1}, z_{2}\right)$ on $D^{2} \times S^{1}$ and $t\left(z_{1}, \rho z_{2}\right)=\left(z_{1}, \rho z_{2}\right)$ on $S^{1} \times D^{2}$. It is checked that $t$ is well-defined and $t h_{1}=h_{2}^{n-1} t$, where $h_{1}=h(a, c, b, d)$ and $h_{2}=$ $h(-a, c,-b, d)$. For (2), define $t$ of $L(a, c, b, d)$ to $L(a,-c, b,-d)$ by $t\left(\rho z_{1}, z_{2}\right)=\left(\rho z_{1}, z_{2}^{-1}\right)$ on $D^{2} \times S^{1}$ and $t\left(z_{1}, \rho z_{2}\right)=\left(z_{1}, \rho z_{2}\right)$ on $S^{1} \times D^{2}$. One may check that $t$ is a well defined equivalence.

By the above lemma, we may assume that $a=p$ and $\left|\begin{array}{l}a \\ b\end{array} d\right|=1$.

LEMMA 3.9. For any integers $b, c, d$ with $p d-c b=1$, 1.

(1) $h(p, c, b, d) \sim h\left(p, c^{\prime}, b, d^{\prime}\right)$ for any integers $c^{\prime}$ and $d^{\prime}$ with $p d^{\prime}-c^{\prime} b=$

(2) $h(p, c, b, d) \sim h(p, c, b+m p, d+m c)$.

(3) $h(p, c, b, d) \sim h(p,-c,-b, d)$.

Proof. Let $L_{1}=L(p, b, c, d)$ and $L_{2}$ is the space corresponding to the weakly equivalent homeomorphism claimed in $(i), i=1,2,3$. In (1), since $p d-b c=1=p d^{\prime}-b c^{\prime}, c^{\prime}=c+m p, d^{\prime}=d+m b$ for some integer $m$. Define $t: L_{1} \rightarrow L_{2}$ by

$$
\begin{array}{ll}
t\left(\rho z_{1}, z_{2}\right)=\left(\rho z_{1} z_{2}^{-m}, z_{2}\right) & \text { on } D^{2} \times S^{1}, \\
t\left(z_{1}, \rho z_{2}\right)=\left(z_{1}, \rho z_{2}\right) & \text { on } S^{1} \times D^{2} .
\end{array}
$$

For (2), define $t: L_{1} \rightarrow L_{2}$ by

$$
\begin{array}{ll}
t\left(\rho z_{1}, z_{2}\right)=\left(\rho z_{1}, z_{2}\right) & \text { on } D^{2} \times S^{1}, \\
t\left(z_{1}, \rho z_{2}\right)=\left(z_{1}, \rho z_{2} z_{1}^{m}\right) & \text { on } S^{1} \times D^{2} .
\end{array}
$$

Notice that $m \equiv 0 \bmod n$ by Lemma 3.5. For (3), define $t: L_{1} \rightarrow L_{2}$ by

$$
\begin{array}{ll}
t\left(\rho z_{1}, z_{2}\right)=\left(\rho z_{1}, z_{2}^{-1}\right) & \text { on } D^{2} \times S^{1} \\
t\left(z_{1}, \rho z_{2}\right)=\left(z_{1}, \rho z_{2}^{-1}\right) & \text { on } S^{1} \times D^{2} .
\end{array}
$$

It is checked that those $t$ are well-defined equivalences.

Now we are in a position to prove Theorem $A$.

(3.10). Proof of Theorem A. By (3.7) and Lemma 3.8, we will consider two maps $h_{1}=h(p, c, b, d)$ and $h_{2}=h\left(p, c^{\prime}, b^{\prime}, d^{\prime}\right)$, where $p d-c b=1=$ $p d^{\prime}-c^{\prime} b^{\prime}$. Since $L(p, c, b, d) \approx L(p, b)$ and $L\left(p, c^{\prime}, b^{\prime}, d^{\prime}\right) \approx L\left(p, b^{\prime}\right)$, we see that $b \equiv \pm b^{\prime}$ or $b b^{\prime} \equiv \pm 1 \bmod p$. If $b^{\prime} \equiv \pm b \bmod p$, it follows from Lemma 3.9 that $h_{1} \sim h_{2}$.

Case $1 . b^{2} \equiv \pm 1 \bmod p$ (symmetric case). Then $b \equiv \pm b^{\prime} \bmod p$, and $h_{1} \sim h_{2}$.

Case 2. $b^{2} \neq \pm 1 \bmod p$ (nonsymmetric case). If $b \equiv \pm b^{\prime} \bmod p$, then $h_{1} \sim h_{2}$. Now suppose that $b b^{\prime} \equiv \pm 1 \bmod p$ (note that such $b$ and $b^{\prime}$ exist in this case; see (3.7) and use the fact $(p, n b)=1)$. Then $b \neq \pm b^{\prime} \bmod p$. Notice that if $b b^{\prime \prime} \equiv \pm 1 \bmod p$ for some $b^{\prime \prime}$, then $b^{\prime \prime} \equiv \pm b^{\prime} \bmod p$ (since $b c \equiv \pm 1 \bmod p)$. Therefore, if we show that $h_{1}$ is not weakly equivalent to $h_{2}$, 
then we are done. Suppose that there exists a weak equivalence $t$ between $h_{1}$ and $h_{2}$. Since $h_{2}\left(t\left(D^{2} \times S^{1}\right)\right)=t\left(D^{2} \times S^{1}\right)$ and $t\left(0 \times S^{1}\right)=0 \times S^{1}$, we may assume that $t\left(D^{2} \times S^{1}\right)=D^{2} \times S^{1}$. Consider the following commutative diagram.

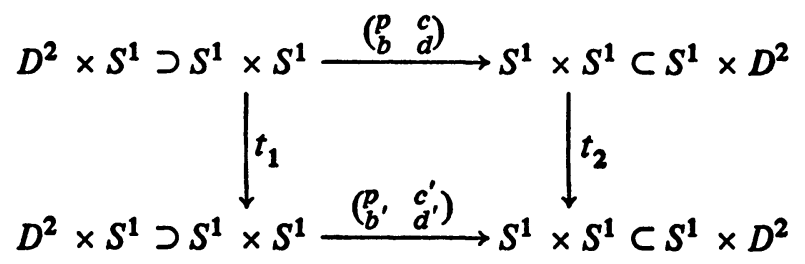

where $t_{1}=t \mid S^{1} \times S^{1} \subset D^{2} \times S^{1}$ and $t_{2}=t \mid S^{1} \times S^{1} \subset S^{1} \times D^{2}$. By the way we defined the generators $(1,0)$ and $(0,1)$ of $\pi_{1}\left(S^{1} \times S^{1}\right)$, we may assume that $t_{1 \#}$ and $t_{2 \#}$ are given by

$$
\left(\begin{array}{cc} 
\pm 1 & k \\
0 & \pm 1
\end{array}\right) \text { and }\left(\begin{array}{cc} 
\pm 1 & 0 \\
k^{\prime} & \pm 1
\end{array}\right)
$$

for some $k, k^{\prime}$, respectively. Therefore, by the above diagram, we see that $b \equiv \pm b^{\prime} \bmod p$, which is a contradiction. This completes the proof of Theorem A.

II. Proof of Theorem B. Fix $(h)$ is a disjoint union of two simple closed curves. Let $F=\operatorname{Fix}(h)$. Let $g: S^{3} \rightarrow L$ be the natural projection. Since $\pi_{1}(L-F)=Z \oplus Z$, and there is an obvious short exact sequence

$$
0 \rightarrow \pi_{1}\left(S^{3}-\tilde{F}\right) \rightarrow Z \oplus Z \rightarrow Z_{p} \rightarrow 0,
$$

$\pi_{1}\left(S^{3}-\tilde{F}\right) \simeq Z \oplus Z$ where $\tilde{F}=g^{-1}(F)$. Therefore $S^{3}-\tilde{F}$ is irreducible (see Theorem 2 of [15]) (of course, $\tilde{F}$ turns out to be a disjoint union of two simple closed curves). Hence, $L-F$ is irreducible. Let $U_{i}(i=1,2)$ be a regular neighborhood of each component of Fix $(h)$ such that $U_{1} \cap U_{2}=\varnothing$. By Stallings [20] (see the proof of Lemma 2.2), we have

LeMMa 3.11. $\mathrm{cl}\left(L-U_{1}-U_{2}\right)$ is homeomorphic to $\partial U_{1} \times I$.

By the above lemma, $L$ may be given as $L=D^{2} \times S^{1} \cup_{k} S^{1} \times D^{2}$ for some attaching map $k$ of $S^{1} \times S^{1}$, where $D^{2} \times S^{1}$ is an invariant regular neighborhood of a component $F_{1}$ of Fix $(h)$ such that $D^{2} \times S^{1} \cap$ Fix $(h)=$ $F_{1}$. Let $h_{1}=h \mid D^{2} \times S^{1}$ and $h_{2}=h \mid S^{1} \times D^{2}$. By Proposition 2.4, we may assume that

$$
L=D^{2} \times S^{1} \cup_{f} S^{1} \times D^{2}
$$

for an appropriate attaching map $f$ of $S^{1} \times S^{1}$ and $h$ is given by 


$$
\begin{aligned}
& e\left(h_{1}\right)\left(\rho z_{1}, z_{2}\right)=\left(\rho \omega z_{1}, z_{2}\right) \text { on } D^{2} \times S^{1} \text { and } \\
& e^{\prime}\left(h_{2}\right)\left(z_{1}, \rho z_{2}\right)=\left(z_{1}, \rho \omega z_{2}\right) \text { on } S^{1} \times D^{2} \text {, }
\end{aligned}
$$

where $\omega=e^{2 \pi i / n}$, and $e, e^{\prime}$ are some automorphisms of $\left\langle h_{1}\right\rangle$ and $\left\langle h_{2}\right\rangle$, respectively. Let $(1,0)$ and $(0,1)$ be the canonical generators of $\pi_{1}\left(S^{1} \times S^{1}\right)$. Let $f_{\#}=\left(\begin{array}{ll}a & c \\ b & d\end{array}\right)$. Then $a= \pm p$ and $\left|\begin{array}{ll}a & c \\ b & d\end{array}\right|= \pm 1$.

Proposition 3.12. $a \equiv 0 \bmod n$.

Proof. The orbit space $L /\langle h\rangle$ may be given as $D^{2} \times S^{1} \cup_{f^{\prime}} S^{1} \times D^{2}$. An easy computation shows that the isotopy class of $f^{\prime}$ is determined by the integers $a / n, b, c$, and $n d$ (cf. the proof of Lemma 3.4).

COROLlaRY 3.13. Let $T$ be a generator of $Z_{2^{n}}$ acting on $P^{3}$. If $\operatorname{Fix}\left(T^{2^{n-1}}\right) \neq$ $0, T^{2^{n-1}}$ is the only element of $Z_{2^{n}}$ with disconnect fixed-point set.

Proof. Since $T^{2^{n-1}}$ is an involution, we see that if $\operatorname{Fix}\left(T^{2^{n-1}}\right) \neq \varnothing$, it is a disjoint union of two simple closed curves [5]. If $\operatorname{Fix}\left(T^{i}\right)$ is not connected for some $i \neq 2^{n-1}, \operatorname{Fix}\left(T^{2^{n-2}}\right)$ is not connected. Let $T^{\prime}=T^{2^{n-2}}$. Then the period of $T^{\prime}$ is 4. By (3.3) and Proposition 3.12, we arrive at a contradiction.

Define a homeomorphism $h_{0}$ on $L_{0}=L(a, c, b, d)$ by

$$
\begin{array}{ll}
h_{0}\left(\rho z_{1}, z_{2}\right)=\left(\rho \omega z_{1}, z_{2}\right) & \text { on } D^{2} \times S^{1} \text { and } \\
h_{0}\left(z_{1}, \rho z_{2}\right)=\left(z_{1}, \rho \omega^{b} z_{2}\right) & \text { on } S^{1} \times D^{2},
\end{array}
$$

where $\omega=e^{2 \pi i / n}$ (see (3.5) for the definition of $L_{0}$ ). Denote $h_{0}$ by $h(a, c, b, d)$. By Proposition 3.12, $h_{0}$ is a well-defined homeomorphism of period $n$. By the proof of Proposition 3.12 and in a similar way to the proof of Lemma 3.6, we claim that $h$ and $h_{0}$ are weakly equivalent. For, in the proof of Lemma $3.6, t^{\prime}$ may be chosen in a way that $t^{\prime}\left(\rho z_{1}, z_{2}\right)=\left(\rho z_{1}, z_{2}\right)$ on $D^{2} \times S^{1}$ and $\left(D^{2}-0\right) \times S^{1}$ is a deformation retract of $L-\operatorname{Fix}(h)$ (and $L_{0}-$ Fix $\left(h_{0}\right)$ ). Now the claim follows easily. Therefore, we reduced our classification problem to that of all homeomorphisms $h(a, c, b, d)$ for various possible $a, c, b, d$, where $a= \pm p$ and $a d-c b= \pm 1$.

LEMMA 3.14. The same statements as in Lemma 3.8 and Lemma 3.9 are also true. Furthermore, $h_{1}=h(p, c, b, d) \sim h_{2}=h(p,-b,-c, d)$ with pd$b c=1$.

Proof. As the proofs are similar to those of Lemma 3.9 and Lemma 3.10, we omit the proofs except the last claim (for the proof of the second statement of Lemma 3.10, use the fact that $p \equiv 0 \bmod n)$. For the last claim, define $t$ of $L(a, c, b, d)$ to $L(a,-b,-c, d)$ by $t\left(\rho z_{1}, z_{2}\right)=\left(z_{2}, \rho z_{1}\right)$ on $D^{2} \times S^{1}$ and $t\left(z_{1}, \rho z_{2}\right)=\left(\rho z_{2}, z_{1}\right)$ on $S^{1} \times D^{2}$ such that $t\left(D^{2} \times S^{1}\right)=S^{1}$ $\times D^{2}$. It is checked that $t$ is well defined and $t h_{1}=h_{2}^{b} t$ (use the fact that $p \equiv 0 \bmod n$ and $p d-c b=1)$. 
(3.15). Proof of Theorem B. By Lemma 3.14, it is enough to consider two homeomorphisms $h_{1}=h(p, c, b, d)$ and $h_{2}=h\left(p, c^{\prime}, b^{\prime}, d^{\prime}\right)$ with $p d-c b=$ $1=p d^{\prime}-c^{\prime} b^{\prime}$. Since $L(p, c, b, d) \approx L\left(p, c^{\prime}, b^{\prime}, d^{\prime}\right), b \equiv \pm b^{\prime}$ or $b b^{\prime} \equiv \pm 1$ $\bmod p$. If $b \equiv \pm b^{\prime} \bmod p, b= \pm b^{\prime}+m p$ for some $m$. By Lemma 3.14, $h_{1} \sim h_{2}$. If $b b^{\prime} \equiv \pm 1 \bmod p$, we see that $b^{\prime} \equiv \pm c \bmod p$ (note that $p d-c b$ $=1$ ). Again by Lemma 3.14, $h_{1} \sim h_{2}$. This completes the proof. (For existence of such $h$, let $s, r$ be integers such that $p s-q r=1$. Then since $p \equiv 0$ $\bmod n$, we can define a map $h^{\prime}=h(p, r, q, s)$. Let $t$ be a homeomorphism of $L(p, q)$ to $L(p, r, q, s)$. Then $t^{-1} h^{\prime} t$ is the homeomorphism as desired.)

4. $Z_{4}$-actions on $L(p, q)$. In the present section we classify all orientationpreserving nonfree $Z_{4}$-actions on $L=L(p, q)$.

Throughout this section, $h$ will always denote an orientation-preserving homeomorphism of period 4 on $L$ with $\operatorname{Fix}\left(h^{2}\right) \neq \varnothing$. A subset $S$ of $L$ will be called $h^{i}$-invariant if $S$ is invariant under $h^{i}$. We denote the number of components of $\operatorname{Fix}\left(h^{i}\right)$ by $c\left(\operatorname{Fix}\left(h^{i}\right)\right)$. Notice that $c\left(\operatorname{Fix}\left(h^{i}\right)\right)<2($ see $\$ 3)$.

It is natural to consider the following five possible cases:

(1) $c(\operatorname{Fix}(h))=0, c\left(\operatorname{Fix}\left(h^{2}\right)\right)=2$.

(2) $c(\operatorname{Fix}(h))=0, c\left(\operatorname{Fix}\left(h^{2}\right)\right)=1$.

(3) $c(\operatorname{Fix}(h))=1, c\left(\operatorname{Fix}\left(h^{2}\right)\right)=2$.

(4) $c(\operatorname{Fix}(h))=1, c\left(\operatorname{Fix}\left(h^{2}\right)\right)=1$.

(5) $c(\operatorname{Fix}(h))=2, c\left(\operatorname{Fix}\left(h^{2}\right)\right)=2$.

Since cases (4) and (5) are special types of Theorems A and B (note that Lemma 3.4 is still valid in case (4), and $p$ is odd in this case), we just investigate cases (1), (2), and (3). In the following we divide the section into three parts, $\mathrm{A}, \mathrm{B}$, and $\mathrm{C}$, according to the three cases.

A. We assume that $c(\operatorname{Fix}(h))=0$ and $c\left(\operatorname{Fix}\left(h^{2}\right)\right)=2$. Let $F_{1}$ and $F_{2}$ be the components of Fix $\left(h^{2}\right)$. Since $\operatorname{Fix}(h)=\varnothing$, we see that either $h\left(F_{i}\right)=F_{i}$ $(i=1,2)$ or $h\left(F_{1}\right)=F_{2}$. The case where $h\left(F_{i}\right)=F_{i}$ and the case where $h\left(F_{1}\right)=F_{2}$ will be studied in I and II, following separately.

I. $h\left(F_{1}\right)=F_{2}$.

LeMma 4.1. There exists an h-invariant torus $S$ embedded in $L$ such that $S$ separates $L$ into two solid tori.

Proof. There exists an $h^{2}$-invariant regular neighborhood $N$ of $F_{1}$ in $L$ such that $N \cap h(N)=\varnothing$. Since $h^{2}$ is a sense-preserving involution (see (3.3)), $\operatorname{cl}(L-N)$ is homeomorphic to $S^{1} \times D^{2}$, and $\left(\operatorname{cl}(L-N), F_{2}\right) \approx\left(S^{1} \times\right.$ $\left.D^{2}, S^{1} \times 0\right)$ (see (2.5)). Obviously $\operatorname{cl}(L-N-h(N))$ is homeomorphic to $S^{1} \times S^{1} \times I$. Let $g$ be the orbit map generated by $h^{2}$. Let $U=\operatorname{cl}(L-N-$ $h(N)$ ). Then $g(U)$ is homeomorphic to $S^{1} \times S^{1} \times I$ (see the proof of Lemma 2.2). Let $\bar{h}$ be the homeomorphism on $L /\left\langle h^{2}\right\rangle$ induced by $h$. Then $\bar{h}$ is a free 
involution and $\bar{h}$ interchanges the two boundary components of $g(U)$. Therefore, there exists an $\bar{h}$-invariant torus $A$ in $\operatorname{Int}(g(U))$ such that $A$ is parallel to each component of $\partial g(U)$ (i.e., $(g(U), A) \approx\left(S^{1} \times S^{1} \times I, S^{1} \times S^{1} \times\right.$ $\left.\left\{\frac{1}{2}\right\}\right)$ ) (see [22]). Since $g^{-1}(A)$ is invariant under $h$ and $h^{2}$, we see that $g^{-1}(A)$ is connected (note that $g$ is induced by $h^{2}$ ). Therefore, $g^{-1}(A)$ is a torus and it is parallel to each component of $\partial U$. Thus, $g^{-1}(A)$ is the torus as desired.

(4.2). By the proof of Lemma 4.1, $L$ may be given as

$$
L=D^{2} \times S^{1} \cup_{k^{\prime}} S^{1} \times S^{1} \times[-1,1] \cup_{k} S^{1} \times D^{2},
$$

where $k$ ( $k^{\prime}$, resp.) is an attaching map of $S^{1} \times S^{1} \times\{1\}\left(S^{1} \times S^{1} \times\{-1\}\right.$, resp.) to $S^{1} \times S^{1} \subset S^{1} \times D^{2}\left(S^{1} \times S^{1} \subset D^{2} \times S^{1}\right.$, resp.), so that $h$ interchanges $D^{2} \times S^{1}$ and $S^{1} \times D^{2}$, and $S^{1} \times S^{1} \times[-1,0]$ and $S^{1} \times S^{1} \times$ $[0,1]$.

LEMMA 4.3. Let $T$ be an orientation-preserving homeomorphism of period 4 on $S^{1} \times S^{1} \times[-1,1]$ such that $\operatorname{Fix}\left(T^{2}\right)=\varnothing$ and $T$ interchanges the two boundary components. Then $T$ is weakly equivalent to a homeomorphism $T^{\prime}$ given by either $T^{\prime}\left(z_{1}, z_{2}, t\right)=\left(i z_{1}, \bar{z}_{2},-t\right)$ or $T^{\prime}\left(z_{1}, z_{2}, t\right)=\left(i z_{1} z_{2}, \bar{z}_{2},-t\right)$ for each $\left(z_{1}, z_{2}, t\right) \in S^{1} \times S^{1} \times[-1,1]$.

Proof. By the proof of Lemma 4.1, we may assume that $T\left(S^{1} \times S^{1} \times\right.$ $\{0\})=S^{1} \times S^{1} \times\{0\}$. Since $S^{1} \times S^{1} \times[0,1]$ is $h^{2}$-invariant, there exists a deformation of the product fibering $S^{1} \times S^{1} \times[0,1]$, constant on $S^{1} \times S^{1}$ $\times\{0\}$, after which $h^{2}(x, t)=(f(x), t)$ for each $x \in S^{1} \times S^{1}$ and $t \in[0,1]$ where $f$ is an involution of $S^{1} \times S^{1}$ (see [22]). Since the deformation is chosen constant on $S^{1} \times S^{1} \times\{0\}$ and $h$ interchanges $S^{1} \times S^{1} \times[-1,0]$ and $S^{1} \times S^{1} \times[0,1]$, one can define a product structure on $S^{1} \times S^{1} \times$ $[-1,1]$ such that $h(x, t)=(\phi(x),-t)$ for each $x \in S^{1} \times S^{1}$ and $t \in$ $[-1,1]$, where $\phi$ is an orientation-reversing homeomorphism of period 4 on $S^{1} \times S^{1}$ and $\phi^{2}=f$.

Now if we show that $\phi$ is conjugate to a map $\phi_{1}$ or $\phi_{2}$ where $\phi_{1}\left(z_{1}, z_{2}\right)=$ $\left(i z_{1}, \bar{z}_{2}\right)$ and $\phi_{2}\left(z_{1}, z_{2}\right)=\left(i z_{1} z_{2}, \bar{z}_{2}\right)$ for each $\left(z_{1}, z_{2}\right) \in S^{1} \times S^{1}$, then we are done. Notice that the orbit space $S^{1} \times S^{1} /\langle\phi\rangle$ is the Klein bottle $K$. Consider an exact sequence $\pi_{1}(K) \rightarrow Z_{4} \rightarrow 0$, where $\pi_{1}(K)=\left\{x, y \mid x y x^{-1} y\right\}$ and $Z_{4}=\langle a\rangle$. Since the sequence can be factored as

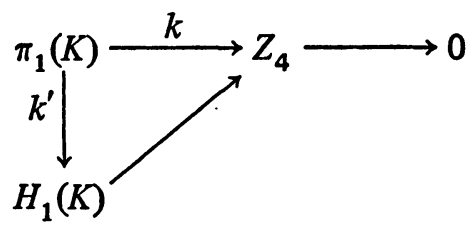

we have $k(x)=a$ or $a^{-1}$, and $k(y)=a^{2}$ or 1 . Therefore, we see that $\operatorname{ker}(k)$ is 
given by either $A_{1}=\left[x^{4}, y\right]$ or $A_{2}=\left[x^{4}, x^{2} y\right]$, where $\left[x^{4}, y\right]$ is the smallest subgroup of $\pi_{1}(K)$ generated by $\left\{x^{4}, y\right\}$ (note that $x^{2}$ and $y$ commute in $\pi_{1}(K)$, and $A_{1}, A_{2}$ are normal subgroups). Since $k^{\prime}\left(A_{1}\right)$ has an element of order 2 but $k^{\prime}\left(A_{2}\right)$ does not, $A_{1}$ is not automorphic to $A_{2}$. Now it is not difficult to see that $g_{j \neq}\left(\pi_{1}\left(S^{1} \times S^{1}\right)\right)(j=1,2)$ is automorphic to the subgroup $A_{j}$ where $g_{j}$ is the orbit map generated by the map $\phi_{j}$. This completes the proof.

By Proposition 2.4, (4.2) and Lemma 4.3, we may assume that

$$
L=D^{2} \times S^{1} \cup_{\alpha} S^{1} \times S^{1} \times[-1,1] \cup_{\beta} S^{1} \times D^{2}
$$

for appropriate attaching maps $\alpha$ and $\beta$ (we shall denote this $L$ by $L[\alpha, \beta]$ ), and $S^{1} \times S^{1} \times[-1,1]$ is $h$-invariant and $h$ interchanges $S^{1} \times D^{2}$ and $D^{2} \times S^{1}$ such that

$$
\begin{aligned}
& h^{2}\left(\rho z_{1}, z_{2}\right)=\left(-\rho z_{1}, z_{2}\right) \text { on } D^{2} \times S^{1}, \\
& h^{2}\left(z_{1}, \rho z_{2}\right)=\left(z_{1},-\rho z_{2}\right) \text { on } S^{1} \times D^{2}, \text { and } \\
& h\left(z_{1}, z_{2}, t\right)=\left(i z_{1}, \bar{z}_{2},-t\right) \quad \text { on } S^{1} \times S^{1} \times[-1,1] \\
& \quad\left(\text { or } h\left(z_{1}, z_{2}, t\right)=\left(i z_{1} z_{2}, \bar{z}_{2},-t\right)\right) .
\end{aligned}
$$

If $h$ is defined on $L[\alpha, \beta]$ in the above manner and $h\left(z_{1}, z_{2}, t\right)=\left(i z_{1}, \bar{z}_{2}\right.$, $-t)$ on $S^{1} \times S^{1} \times[-1,1]\left(h\left(z_{1}, z_{2}, t\right)=\left(i z_{1} z_{2}, \bar{z}_{2},-t\right)\right.$, resp.), this $h$ will be said to be of type $(\alpha, \beta)$ (type $(\alpha, \beta)^{*}$, resp.). Any two homeomorphisms of types $(\alpha, \beta)$ and $\left(\alpha^{\prime}, \beta^{\prime}\right)^{*}$ are not conjugate (see the proof of Lemma 4.3). In fact, in this case $L[\alpha, \beta]$ is not even homeomorphic to $L\left[\alpha^{\prime}, \beta^{\prime}\right]$ (see Lemma 4.11).

Let $(1,0)$ and $(0,1)$ be the canonical generators of $\pi_{1}\left(S^{1} \times S^{1}\right)$. Let $\left(\begin{array}{ll}a & c \\ b & d\end{array}\right)$ be the matrix of $\beta$. Then

$$
\left|\begin{array}{ll}
a & c \\
b & d
\end{array}\right|= \pm 1
$$

The proof of Lemma 4.5 is based on homeomorphisms of type $(\alpha, \beta)$. A similar proof goes through for the case of type $(\alpha, \beta)^{*}$ (in this case one can observe that the homomorphism $f_{i \#}$ in the proof may be given by $f_{i \#}[(1,0)]=$ $(2,0)$ and $f_{i \#}[(0,1)]=(1,1)$, and the matrix of $\beta_{i}^{\prime}$ may be given by

$$
\left(\begin{array}{cc}
a / 2 & c-a / 2 \\
b & 2 d-b
\end{array}\right)
$$

LEMMA 4.4. $a$ is even.

Proof. See the proof of Lemma 4.5.

Lemma 4.5. Suppose that $h_{i}(i=1,2)$ is of type $\left(\alpha_{i}, \beta_{i}\right)\left(\right.$ or $\left.\left(\alpha_{i}, \beta_{i}\right)^{*}\right)$. If the 
matrices of $\beta_{1}$ and $\beta_{2}$ are the same, then $L\left[\alpha_{1}, \beta_{1}\right] \approx L\left[\alpha_{2}, \beta_{2}\right]$, and $h_{1}$ is weakly equivalent to $h_{2}$.

Proof. Let $g_{i}$ be the orbit map generated by $\left\langle h_{i}^{2}\right\rangle$. We may assume that $L\left[\alpha_{i}, \beta_{i}\right] /\left\langle h_{i}^{2}\right\rangle$ is given as $L\left[\alpha_{i}^{\prime}, \beta_{i}^{\prime}\right]$ for some $\alpha_{i}^{\prime}, \beta_{i}^{\prime}$ such that

$$
g_{i}\left(S^{1} \times S^{1} \times[-1,1]\right)=S^{1} \times S^{1} \times[-1,1]
$$

and

$$
g_{i}\left(S^{1} \times D^{2}\right)=S^{1} \times D^{2}
$$

(and $\left.g_{i}\left(S^{1} \times 0\right)=S^{1} \times 0\right)$. Let

$$
f_{i}=g_{i} \mid S^{1} \times S^{1} \times\{1\} \text { and } f_{i}^{\prime}=g_{i} \mid S^{1} \times S^{1} \subset S^{1} \times D^{2} .
$$

Then we may further assume that $f_{i \#}[(1,0)]=(2,0)$ and $f_{i \#}[(0,1)]=(0,1)$, and $f_{i \#}^{\prime}[(1,0)]=(1,0)$ and $f_{i \#}^{\prime}[(0,1)]=(0,2)$. Let $\left(\begin{array}{cc}a & c \\ b & d\end{array}\right)$ be the matrix of $\beta_{i}$. A simple computation shows that the matrix of $\beta_{i}^{\prime}$ may be given by $\left(\begin{array}{cc}a / 2 & c \\ b & 2 d\end{array}\right)$. Let $\bar{h}_{i}$ be the free involution on $L\left[\alpha_{i}^{\prime}, \beta_{i}^{\prime}\right]$ induced by $h_{i}$. We may assume that

$$
\begin{gathered}
\overline{h_{i}}\left(S^{1} \times D^{2}\right)=D^{2} \times S^{1} \quad\left(\text { and } \bar{h}_{i}\left(S^{1} \times 0\right)=0 \times S^{1}\right), \\
\bar{h}_{i}\left(S^{1} \times S^{1} \times[0,1]\right)=S^{1} \times S^{1} \times[-1,0] \text { and } \\
\overline{h_{i}}\left|S^{1} \times S^{1} \times[-1,1]=\bar{h}_{2}\right| S^{1} \times S^{1} \times[-1,1]
\end{gathered}
$$

(see the definition of $h_{i}$ ).

We will define a homeomorphism $t$ of $L\left[\alpha_{1}^{\prime}, \beta_{1}^{\prime}\right]$ to $L\left[\alpha_{2}^{\prime}, \beta_{2}^{\prime}\right]$ such that $t \bar{h}_{1}=\bar{h}_{2} t$, and $t\left(0 \times S^{1}\right)=0 \times S^{1}$ and $t\left(S^{1} \times 0\right)=S^{1} \times 0$, where $0 \times S^{1}$ $\subset D^{2} \times S^{1}$ and $S^{1} \times 0 \subset S^{1} \times D^{2}$. Since $\beta_{1}^{\prime}$ and $\beta_{2}^{\prime}$ are isotopic (see (3.5)), there exists $t_{1}$ of $S^{1} \times S^{1} \times[0,1] \cup{\beta_{1}^{\prime}}^{1} \times D^{2}$ to $S^{1} \times S^{1} \times[0,1] \cup{\beta_{2}}^{\prime} S^{1} \times$ $D^{2}$ such that $t_{1}$ sends $S^{1} \times S^{1} \times[0,1]$ to $S^{1} \times S^{1} \times[0,1]$ by $t_{1}\left(z_{1}, z_{2}, t\right)=$ $\left(z_{1}, z_{2}, t\right)$, and $t_{1}\left(0 \times S^{1}\right)=0 \times S^{1}$. Let $t$ be the homeomorphism of $L\left[\alpha_{1}^{\prime}, \beta_{1}^{\prime}\right]$ to $L\left[\alpha_{2}^{\prime}, \beta_{2}^{\prime}\right]$ defined by $t=t_{1}$ on $S^{1} \times S^{1} \times[0,1] \cup_{\beta_{1}^{\prime}} S^{1} \times D^{2}$ and $t=$ $\overline{h_{2}} t_{1} \bar{h}_{1}$ on $D^{2} \times S^{1} \cup_{\alpha_{1}^{\prime}} S^{1} \times S^{1} \times[-1,0]$. This $t$ is well defined (recall that $\left.\bar{h}_{1}\left|S^{1} \times S^{1} \times\{0\}=\bar{h}_{2}\right| S^{1} \times S^{1} \times\{0\}\right)$, and it is the map as desired.

Let $t^{\prime}=t \mid S^{1} \times S^{1} \times\{1\}$. Then we see that $t_{\#}^{\prime} f_{1 \#}=f_{2 \# \text {. Since }} S^{1} \times S^{1}$ $\times\{1\}$ is a deformation retract of

$$
\left(D^{2}-0\right) \times S^{1} \cup_{\alpha_{i}} S^{1} \times S^{1} \times[-1,1] \cup_{\beta_{i}} S^{1} \times\left(D^{2}-0\right)
$$

(we denote this by $L^{\prime}\left[\alpha_{i}, \beta_{i}\right]$ ), it follows from the lifting theorem that there exists a homeomorphism $\bar{t}$ of $L^{\prime}\left[\alpha_{1}, \beta_{1}\right]$ to $L^{\prime}\left[\alpha_{2}, \beta_{2}\right]$ such that $g_{2} \bar{t}=\operatorname{tg} g_{1}$ on $L^{\prime}\left[\alpha_{1}, \beta_{1}\right]$. Since Fix $\left(h^{2}\right)=0 \times S^{1} \cup S^{1} \times 0 \subset L\left[\alpha_{i}, \beta_{i}\right]$, and $t\left(0 \times S^{1}\right)=0$ $\times S^{1}$ and $t\left(S^{1} \times 0\right)=S^{1} \times 0$ in $L\left[\alpha_{i}^{\prime}, \beta_{i}^{\prime}\right]$, one can extend $t$ to a homeomorphism $\overline{t^{\prime}}$ of $L\left[\alpha_{1}, \beta_{1}\right]$ to $L\left[\alpha_{2}, \beta_{2}\right]$ such that $g_{2} \bar{t}^{\prime}=t g_{1}$. Therefore, we see that $h_{1}$ and $h_{2}$ are weakly equivalent. This completes the proof.

(4.6). According to Lemma 4.5, if $h$ if of type $(\alpha, \beta)$ (or $\left.(\alpha, \beta)^{*}\right)$, then $h$ is 
completely determined (up to weak equivalence) by the matrix of $\beta$. Therefore, we may assume that

$$
L=D^{2} \times S^{1} \cup_{\alpha} S^{1} \times S^{1} \times[-1,1] \cup_{\beta} S^{1} \times D^{2}
$$

where $\beta\left(z_{1}, z_{2}, 1\right)=\left(z_{1}^{a} z_{2}^{c}, z_{1}^{b} z_{2}^{d}\right)$ and $\alpha\left(z_{1}, z_{2},-1\right)=\left(z_{1}^{b} z_{2}^{-d}, z_{1}^{a} z_{2}^{-c}\right)$ (or $\alpha\left(z_{1}, z_{2},-1\right)=\left(z_{1}^{b} z_{2}^{b-d}, z_{1}^{a_{2}} z_{2}^{a-c}\right)$ for $\left.(\alpha, \beta)^{*}\right)$, and $h$ is defined by

$$
\begin{aligned}
& h\left(\rho z_{1}, z_{2}\right)=\left(i{ }^{a} z_{2}, \rho i^{b} z_{1}\right) \text { on } D^{2} \times S^{1}, \\
& h\left(z_{1}, z_{2}, t\right)=\left(i z_{1}, z_{2}^{-1},-t\right) \text { on } S^{1} \times S^{1} \times[-1,1] \\
& \quad\left(\text { or } h\left(z_{1}, z_{2}, t\right)=\left(i z_{1} z_{2}, z_{2}^{-1},-t\right) \text { for }(\alpha, \beta)^{*}\right) \text {, and } \\
& h\left(z_{1}, \rho z_{2}\right)=\left(\rho i i_{2}, i^{a} z_{1}\right) \text { on } S^{1} \times D^{2},
\end{aligned}
$$

where $h\left(D^{2} \times S^{1}\right)=S^{1} \times D^{2}$ and $S^{1} \times S^{1} \times[-1,1]$ is $h$-invariant. It is checked that this $h$ is well-defined and of period 4 (recall that $a$ is even (Lemma 4.4), and therefore $b$ is odd). The differences between the two new homeomorphisms $h$ of types $(\alpha, \beta)$ and $(\alpha, \beta)^{*}$ have been indicated by inserting parentheses. We shall denote this homeomorphism $h$ of type $(\alpha, \beta)$ (type $(\alpha, \beta)^{*}$, resp.) by $h^{\dagger}[a, c, b, d]\left(h^{*}[a, c, b, d]\right.$, resp.) and the corresponding $L$ by $L^{\dagger}[a, c, b, d]\left(L^{*}[a, c, b, d]\right.$, resp.). We let $h[a, c, b, d]$ denote either $h^{\dagger}[a, c, b, d]$ or $h^{*}[a, c, b, d]$. In what follows $h[a, c, b, d] \sim$ $h\left[a^{\prime}, c^{\prime}, b^{\prime}, d^{\prime}\right]$ means $h^{\dagger}[a, c, b, d] \sim h^{\dagger}\left[a^{\prime}, c^{\prime}, b^{\prime}, d^{\prime}\right]$ and $h^{*}[a, c, b, d] \sim$ $h^{*}\left[a^{\prime}, c^{\prime}, b^{\prime}, d^{\prime}\right]$.

LEMMA 4.7. (1) $h[a, c, b, d] \sim h[-a,-c, b, d]$.

(2) $h[a, c, b, d] \sim h[a, c,-b,-d]$.

Proof. For (1), define $t$ of $L[a, c, b, d]$ to $L[-a, c,-b, d]$ by $t\left(\rho z_{1}, z_{2}\right)=$ $\left(\rho z_{1}, z_{2}^{-1}\right)$ on $D^{2} \times S^{1}, t\left(z_{1}, z_{2}, t^{\prime}\right)=\left(z_{1}, z_{2}, t^{\prime}\right)$ on $S^{1} \times S^{1} \times[-1,1]$, and $t\left(z_{1}, \rho z_{2}\right)=\left(z_{1}^{-1}, \rho z_{2}\right)$ on $S^{1} \times D^{2}$. For (2), define $t$ of $L[a, c, b, d]$ to $L[a,-c, b,-d]$ by $t\left(\rho z_{1}, z_{2}\right)=\left(\rho z_{1}^{-1}, z_{2}\right)$ on $D^{2} \times S^{1}, t\left(z_{1}, z_{2}, t^{\prime}\right)=$ $\left(z_{1}, z_{2}, t^{\prime}\right)$ on $S^{1} \times S^{1} \times[-1,1]$, and $t\left(z_{1}, \rho z_{2}\right)=\left(z_{1}, \rho z_{2}^{-1}\right)$ on $S^{1} \times D^{2}$. It is checked that these $t$ 's are well-defined equivalences.

LEMMA 4.8. (1) $h^{\dagger}[a, c, b, d] \sim h^{\dagger}[a,-c,-b, d]$ and (2) $h[a, c, b, d] \sim$ $h\left[a, c, b^{\prime}, d^{\prime}\right]$, where $a d-c b=a d^{\prime}-c b^{\prime}=1$.

Proof. For (1), define $t$ of $L[a, c, b, d]$ to $L[a,-c,-b, d]$ by $t\left(\rho z_{1}, z_{2}\right)$ $=\left(\rho z_{1}^{-1}, z_{2}\right)$ on $D^{2} \times S^{1}, t\left(z_{1}, z_{2}, t^{\prime}\right)=\left(z_{1}, z_{2}^{-1}, t^{\prime}\right)$ on $S^{1} \times S^{1} \times[-1,1]$, and $t\left(z_{1}, \rho z_{2}\right)=\left(z_{1}, \rho z_{2}^{-1}\right)$ on $S^{1} \times D^{2}$. In (2), since $a d-c b=a d^{\prime}-c b^{\prime}$ we see that $d^{\prime}=d-m c$ and $b^{\prime}=b-m a$ for some $m$. Define $t$ of $L[a, c, b, d]$ to $L\left[a, c, b^{\prime}, d^{\prime}\right]$ by $t\left(\rho z_{1}, z_{2}\right)=\left(\rho z_{1} z_{2}^{-m}, z_{2}\right)$ on $D^{2} \times S^{1}, t\left(z_{1}, z_{2}, t^{\prime}\right)=$ $\left(z_{1}, z_{2}, t^{\prime}\right)$ on $S^{1} \times S^{1} \times[-1,1]$, and $t\left(z_{1}, \rho z_{2}\right)=\left(z_{1}, \rho z_{2} z_{1}^{-m}\right)$ on $S^{1} \times D^{2}$. It is checked that these $t$ 's are well-defined equivalences. 
LEMMA 4.9. (1) $L^{\dagger}=L^{\dagger}[a, c, b, d]$ is homeomorphic to $L(2 a c, a d+b c)$. (2) $L^{*}=L^{*}[a, c, b, d]$ is homeomorphic to $L\left(2 a c-a^{2}, a d+b c-a b\right)$. (3) $L^{\dagger}$ and $L^{*}$ are symmetric.

Proof. Let $\alpha, \beta$ be the maps in (4.6). Then $L$ may be viewed as $D^{2} \times S^{1}$ $\cup_{\beta \alpha^{-1}} S^{1} \times D^{2}$ in the obvious way. The result follows from the matrix of $\beta \alpha^{-1}$ and (3.5).

One can observe the following by an elementary argument, and we omit the proof.

Lemma 4.10. Let $t, s, t^{\prime}, s^{\prime}$ be integers such that $t s= \pm t^{\prime} s^{\prime}$ and $(t, s)=1=$ $\left(t^{\prime}, s^{\prime}\right)$. If $t w+v s \equiv \pm\left(t^{\prime} w^{\prime}+v^{\prime} s^{\prime}\right) \bmod 2 t s$ where $t w-v s=1=t^{\prime} w^{\prime}-$ $v^{\prime} s^{\prime}$, then either $t= \pm t^{\prime}$ or $t= \pm s^{\prime}$.

LEMMA 4.11. Let $a$ and $a^{\prime}$ be even integers. Then $L^{\dagger}[a, c, b, d]$ and $L^{*}\left[a^{\prime}, c^{\prime}, b^{\prime}, d^{\prime}\right]$ are not homeomorphic for any possible integers.

Proof. By the proof of Lemma 4.7, we may assume that $a$ and $a^{\prime}$ are positive even integers and $a d-b c=1=a^{\prime} d^{\prime}-b^{\prime} c^{\prime}$. Suppose the contrary that they were homeomorphic. Then, by Lemma 4.9, we see that

$$
\pm a c=a^{\prime}\left(c^{\prime}-a^{\prime} / 2\right)
$$

and

$$
\pm(a d+b c) \equiv a^{\prime}\left(2 d^{\prime}-b^{\prime}\right) / 2+b^{\prime}\left(c^{\prime}-a^{\prime} / 2\right) \bmod 2 a c
$$

(since $L^{*}$ is symmetric). Therefore, it follows from Lemma 4.10 that either $c= \pm a^{\prime} / 2$ and $a= \pm 2\left(c^{\prime}-a^{\prime} / 2\right)$ or $c= \pm\left(c^{\prime}-a^{\prime} / 2\right)$ and $a=a^{\prime}$ (let $t=a / 2, t^{\prime}=a^{\prime} / 2, s=c$, and $\left.s^{\prime}=c^{\prime}-a^{\prime} / 2\right)$. But, in this case an elementary argument shows that these two possibilities cannot occur (using the above relations and the fact that

$$
a d-b c=1=a^{\prime}\left(2 d^{\prime}-b^{\prime}\right) / 2-b^{\prime}\left(c^{\prime}-a^{\prime} / 2\right),
$$

one can see that

$$
\pm(2 a d-1) \equiv 1 \pm b^{\prime} a \text { or } a\left(2 d^{\prime}-b^{\prime}\right)-1 \bmod 2 a c ;
$$

therefore, $a=2$ or $b^{\prime}$ is even, which leads to a contradiction; note that $a$ is divisible by 4 in this case).

LEMMA 4.12. Let $a$ and $a^{\prime}$ be positive even integers and $a d-b c=1=a^{\prime} d^{\prime}$ $-b^{\prime} c^{\prime}$.

(1) $L_{1}=L^{\dagger}[a, c, b, d]$ is homeomorphic to $L_{2}=L^{\dagger}\left[a^{\prime}, c^{\prime}, b^{\prime}, d^{\prime}\right]$ if and only if $a^{\prime}=a$ and $c^{\prime}= \pm c$.

(2) $L_{1}=L^{*}[a, c, b, d]$ is homeomorphic to $L_{2}=L^{*}\left[a^{\prime}, c^{\prime}, b^{\prime}, d^{\prime}\right]$ if and only if one of the following holds: (i) $a^{\prime}=a$ and either $c^{\prime}=c$ or $c^{\prime}=a-c$. (ii) If $2 c-a>0$, then $a^{\prime}=2 c-a$ and either $c^{\prime}=c$ or $c^{\prime}=c-a($ if $a-2 c>0$, then $a^{\prime}=a-2 c$ and either $c^{\prime}=-c$ or $c^{\prime}=a-c$ ). 
Proof. The following argument works for either case of the above two claims. Suppose that $L_{1}$ is homeomorphic to $L_{2}$. Then the results follow from Lemmas 4.9 and 4.10 (see the proof of Lemma 4.11). For the converse statements, it follows from the proofs of Lemmas 4.8 and 4.9.

Now we are in a position to state our conclusion.

THEOREM 4.13. If $c(\operatorname{Fix}(h))=0$ and $c\left(\operatorname{Fix}\left(h^{2}\right)\right)=2$, and $h$ interchanges the two components of $\operatorname{Fix}\left(h^{2}\right)$, then $p \equiv 0 \bmod 4$ and $q$ is one of the following forms;

(1) $q \equiv a d+b c \bmod p$, where $p=2 a c$ ( $a$ even) and $\left|\begin{array}{ll}a & c \\ b & d\end{array}\right|=1$.

(2) $q \equiv a d+b c-(a b / 2) \bmod p$, where $p=2 a c$ (a even) and

$$
\left|\begin{array}{cc}
a & c+a / 2 \\
b & d
\end{array}\right|=1
$$

Up to weak equivalence, there exists exactly one such $h$ on $L(p, q)$ for $p, q$ of form (1), and there exists exactly two such $h$ on $L(p, q)$ for $p, q$ of form (2).

REMARK. The homeomorphic type of $L(p, q)$ does not depend on the choice of $b$ and $d$ (see the proof of Lemma 4.8). For simplicity, in the proof we let the integers $p, q$ of form (1) (form (2), resp.) denote $p_{1}, q_{1}\left(p_{2}, q_{2}\right.$, resp.) Notice that $p_{2} \equiv 0 \bmod 8$. It follows from Lemmas 4.9 and 4.11 that $L\left(p_{1}, q_{1}\right)$ is not homeomorphic to $L\left(p_{2}, q_{2}\right)$ for any possible $p_{1}, q_{1}, p_{2}, q_{2}$.

Proof. The first part of the theorem follows from Lemma 4.4, (4.6), and Lemma 4.9. For existence, let $h=t h^{\prime} t^{-1}$, where $h^{\prime}=h^{\dagger}[a, c, b, d]$ (or $h^{*}[a, c$ $+a / 2, b, d]$ ) and $t$ is a homeomorphism of $L^{\dagger}[a, c, b, d]$ (or $L^{*}[a, c+$ $a / 2, b, d])$ to $L(p, q)$. For the classification of such $h$ 's, since $L\left(p_{1}, q_{1}\right)$ and $L\left(p_{2}, q_{2}\right)$ are not homeomorphic (see the above note), we consider them separately.

Case 1. $L \approx L\left(p_{1}, q_{1}\right)$. It follows from (4.6) and Lemmas 4.7 and 4.12 that it is enough to show that $h^{\dagger}[a, c, b, d] \sim h^{\dagger}\left[a, c^{\prime}, b^{\prime}, d^{\prime}\right]$, where $c^{\prime}= \pm c$ and $a d-b c=1=a d^{\prime}-b^{\prime} c^{\prime}$. One can see that this follows from Lemma 4.8.

Case 2. $L \approx L\left(p_{2}, q_{2}\right)$. By (4.6) and Lemma 4.7, we compare $h_{1}=$ $h^{*}[a, c, b, d]$ with $h_{2}=h^{*}\left[a^{\prime}, c^{\prime}, b^{\prime}, d^{\prime}\right]$, where $a d-b c=1=a^{\prime} d^{\prime}-b^{\prime} c^{\prime}$ and $a, a^{\prime}$ are even. According to Lemma 4.12, we consider the following subcases.

Subcase (i). $a^{\prime}=a$ and either $c^{\prime}=c$ or $c^{\prime}=a-c$. If $c^{\prime}=c$, then $h_{1} \sim h_{2}$ by Lemma 4.8. Now suppose that $c^{\prime}=a-c$. By Lemma 4.8, we may assume that $b^{\prime}=-b$ and $d^{\prime}=d-b$. Define $t$ of $L^{*}[a, c, b, d]$ to $L^{*}[a, a-c,-$ $b, d-b]$ by

$$
\begin{aligned}
& t\left(\rho z_{1}, z_{2}\right)=\left(\rho z_{1}^{-1}, z_{2}\right) \quad \text { on } D^{2} \times S^{1} \text {, } \\
& t\left(z_{1}, z_{2}, t^{\prime}\right)=\left(z_{1} z_{2}, z_{2}^{-1}, t^{\prime}\right) \quad \text { on } S^{1} \times S^{1} \times[-1,1] \text {, and } \\
& t\left(z_{1}, \rho z_{2}\right)=\left(z_{1}, \rho z_{2}^{-1}\right) \quad \text { on } S^{1} \times D^{2} \text {. }
\end{aligned}
$$


It is checked that this $t$ is a well-defined equivalence between $h_{1}$ and $h_{2}$.

Subcase (ii). $a^{\prime}=2 c-a$ and either $c^{\prime}=c$ or $c^{\prime}=c-a$. Since the following argument also works for the case where $a-2 c>0$, we simply assume that $2 c-a>0$. Suppose the contrary that $h_{1}$ were weakly equivalent to $h_{2}$. Then there exists a weak equivalence $t$ of $L_{1}=L^{*}[a, c, b, d]$ to $L_{2}=$ $L^{*}\left[a^{\prime}, c^{\prime}, b^{\prime}, d^{\prime}\right]$. Note that $t\left(0 \times S^{1}\right)=0 \times S^{1}$ or $S^{1} \times 0$ where $0 \times S^{1} \subset$ $D^{2} \times S^{1}$ and $S^{1} \times 0 \subset S^{1} \times D^{2}$. We may assume that $t\left(0 \times S^{1}\right)=0 \times S^{1}$ and $t\left(S^{1} \times 0\right)=S^{1} \times 0$ (since $h_{2}$ interchanges $S^{1} \times 0$ and $\left.0 \times S^{1}\right)$. Since $h_{2} t\left(D^{2} \times S^{1}\right)=t\left(S^{1} \times D^{2}\right)$ and $h_{2} t\left(S^{1} \times D^{2}\right)=t\left(D^{2} \times S^{1}\right)$, we may assume that $t\left(D^{2} \times S^{1}\right)=D^{2} \times S^{1}$ and $t\left(S^{1} \times D^{2}\right)=S^{1} \times D^{2}$. Note that the matrices of $h_{i} \mid S^{1} \times S^{1} \times[-1,1]$ are given by $\left(\begin{array}{ll}1 & 1 \\ 0 & -1\end{array}\right)$. Since the matrix $M_{1}$ of $t \mid S^{1} \times S^{1} \times[-1,1]$ must commute with $\left(\begin{array}{ll}1 & 1 \\ 0 & -1\end{array}\right), M_{1}$ is of the form

$$
\left(\begin{array}{cc}
\varepsilon_{1} & \gamma \\
0 & \varepsilon_{2}
\end{array}\right)
$$

where each $\varepsilon_{i}= \pm 1$, and $\gamma=0$ or \pm 1 (note that $\left|M_{1}\right|= \pm 1$ ). Let $M_{2}$ be the matrix of $t \mid S^{1} \times S^{1} \subset S^{1} \times D^{2}$. Then $M_{2}$ may be given by

$$
\left(\begin{array}{ll}
\varepsilon_{1}^{\prime} & 0 \\
\gamma^{\prime} & \varepsilon_{2}^{\prime}
\end{array}\right)
$$

where each $\varepsilon_{i}^{\prime}= \pm 1$ and $\gamma^{\prime}$ is some integer (see how $(0,1)$ is defined). Since we require that

$$
M_{2}\left(\begin{array}{ll}
a & c \\
b & d
\end{array}\right)=\left(\begin{array}{ll}
a^{\prime} & c^{\prime} \\
b^{\prime} & d^{\prime}
\end{array}\right) M_{1}
$$

we see that $a^{\prime}=a$, which is a contradiction to the fact that $a^{\prime}=2 c-a$ and $(a, c)=1$. Therefore, in this case $h_{1}$ and $h_{2}$ are not weakly equivalent.

Now if we show that $h^{*}\left[2 c-a, c, b^{\prime}, d^{\prime}\right]$ is weakly equivalent to $h^{*}[2 c-$ $\left.a, c-a, b^{\prime \prime}, d^{\prime \prime}\right]$ where $(2 c-a) d^{\prime}-c b^{\prime}=1=(2 c-a) d^{\prime \prime}-(c-a) b^{\prime \prime}$, then we are done. In fact, it follows from Lemma 4.8 and the above subcase (i) that

$$
\begin{aligned}
h^{*}\left[2 c-a, c, b^{\prime}, d^{\prime}\right] & \sim h^{*}[2 c-a, c, b-2 d,-d] \\
& \sim h^{*}[2 c-a, c-a, 2 d-b, d-b] \\
& \sim h^{*}\left[2 c-a, c-a, b^{\prime \prime}, d^{\prime \prime}\right] .
\end{aligned}
$$

This completes the proof.

II. $h_{i}\left(F_{i}\right)=F_{i}$. Now consider the case where $h_{i}\left(F_{i}\right)=F_{i}(i=1,2)$. Let $N$ be an $h$-invariant regular neighborhood of $F_{1}$ such that $N \cap F_{2}=\varnothing$. Then $\operatorname{cl}(L-N) \approx S^{1} \times D^{2}$ (see (3.3)). By Theorem 2.9, we may assume that $L=D^{2} \times S^{1} \cup_{f} S^{1} \times D^{2}$ for an appropriate attaching map $f$ of $S^{1} \times S^{1}$ and $h$ is given by $h_{1}^{j}\left(\rho z_{1}, z_{2}\right)=\left(\rho i z_{1},-z_{2}\right)$ on $D^{2} \times S^{1}$ and $h_{2}^{k}\left(z_{1}, \rho z_{2}\right)=$ $\left(-z_{1}, \rho i z_{2}\right)$ on $S^{1} \times D^{2}$ where $h_{1}=h \mid D^{2} \times S^{1}$ and $h_{2}=h \mid S^{1} \times D^{2}$, and 
$j, k=1$ or 3 . Let $(1,0)$ and $(0,1)$ be the canonical generators of $\pi_{1}\left(S^{1} \times S^{1}\right)$. Let $g_{i}$ be the orbit maps generated by $\left\langle h_{i}\right\rangle(i=1,2)$. Parmetrize the orbit spaces of $\left\langle h_{1}\right\rangle$ and $\left\langle h_{2}\right\rangle$ in terms of $D^{2} \times S^{1}$ and $S^{1} \times D^{2}$, respectively. Let $g_{i}^{\prime}=g_{i} \mid S^{1} \times S^{1}$. By the proof of Theorem 2.9, we may assume that $g_{1 \#}^{\prime}[(1,0)]=(2,0), g_{2 \#}^{\prime}[(0,1)]=(0,2), g_{1 \#}^{\prime}[(0,1)]=\left(r^{\prime}, 2\right)$, and $g_{2 \#}^{\prime}[(1,0)]=$ $(2, r)$ for some odd integers $r^{\prime}, r$. Let $\left(\begin{array}{cc}a & c \\ b & d\end{array}\right)$ be the matrix of $f$.

LEMMA 4.14. $a \equiv 0 \bmod 4$.

Proof. $L /\langle h\rangle$ may be given as

$$
L /\langle h\rangle=D^{2} \times S^{1} \cup_{f^{\prime}} S^{1} \times D^{2}
$$

where $f^{\prime}$ is induced by $f$. A simple computation shows that

$$
\begin{aligned}
& a^{\prime}=a, \quad b^{\prime}=(2 b+a r) / 2, \quad c^{\prime}=\left(2 c-r^{\prime} a\right) / 2, \\
& d^{\prime}=\left(4 d+2 c r-2 r^{\prime} b-a r^{\prime} r\right) / 4,
\end{aligned}
$$

where the matrix of $f^{\prime}$ is

$$
\left(\begin{array}{ll}
a^{\prime} & c^{\prime} \\
b^{\prime} & d^{\prime}
\end{array}\right)
$$

From $b^{\prime}$ and $d^{\prime}$, we see that $a \equiv 0 \bmod 4$.

Define a homeomorphism $h_{0}$ on $L(a, c, b, d)$ (see (3.5) for the notation) by

$$
\begin{array}{ll}
h_{0}\left(\rho z_{1}, z_{2}\right)=\left(\rho i z_{1},-z_{2}\right) & \text { on } D^{2} \times S^{1} \text { and } \\
h_{0}\left(z_{1}, \rho z_{2}\right)=\left(-z_{1}, \rho i^{2 d+b_{2}}\right) & \text { on } S^{1} \times D^{2} .
\end{array}
$$

It is checked that $h_{0}$ is well defined and $h$ is of period 4 as $a d-b c= \pm 1$.

LEMMA 4.15. $h$ is weakly equivalent to $h_{0}$.

Proof. $L /\langle h\rangle$ and $L_{0} /\left\langle h_{0}\right\rangle$ may be given as

$$
D^{2} \times S^{1} \cup_{f} S^{1} \times D^{2} \text { and } D^{2} \times S^{1} \cup_{f_{0}} S^{1} \times D^{2} \text {, }
$$

respectively. Since $f^{\prime}$ and $f_{0}^{\prime}$ are isotopic, there exists a homeomorphism $t$ of $L /\langle h\rangle$ to $L_{0} /\left\langle h_{0}\right\rangle$ such that $t\left(D^{2} \times S^{1}\right)=D^{2} \times S^{1}$, and $t\left(z_{1}, \rho z_{2}\right)=$ $\left(z_{1}, \rho z_{2}\right)$ on $S^{1} \times D^{2}$. Let $g$ and $g_{0}$ be the orbit maps generated by $\langle h| S^{1} \times$ $\left.\left(D^{2}-0\right)\right\rangle$ and $\left\langle h_{0} \mid S^{1} \times\left(D^{2}-0\right)\right\rangle$. We may assume that $g_{\#}[(1,0)]=(2, r)$ and $g_{0 \#}[(1,0)]=(2, r)$ for some odd $r$ (compare $h \mid S^{1} \times D^{2}$ with $h_{0} \mid S^{1} \times$ $\left.D^{2}\right)$. Since $t\left(z_{1}, \rho z_{2}\right)=\left(z_{1}, \rho z_{2}\right)$ on $S^{1} \times D^{2}$, we have $t_{\#}^{\prime} g_{\#}=g_{0 \#}$ where $t^{\prime}=t \mid S^{1} \times\left(D^{2}-0\right)$. Notice that $S^{1} \times\left(D^{2}-0\right)$ is a deformation retract of $L-\operatorname{Fix}\left(h^{2}\right)$ (and $L_{0}-\operatorname{Fix}\left(h_{0}^{2}\right)$ ). Now one may complete the proof in the same manner as in Theorem 2.9.

We now state our claim.

THEOREM 4.16. If $c(\operatorname{Fix}(h))=0$ and $c\left(\operatorname{Fix}\left(h^{2}\right)\right)=2$, and $h$ fixes each component of $\operatorname{Fix}\left(h^{2}\right)$, then $p \equiv 0 \bmod 4$. Up to weak equivalence, there exists 
exactly one such $h$ on each lens space $L(4 m, q)$.

Proof. We denote $h_{0}$ by $h(a, c, b, d)$. By Lemma 4.15 , it is enough to consider all possible homeomorphisms $h(a, c, b, d)$. It is checked that the same statements as in Lemma 3.14 is also true in this case (use the same homeomorphisms $t$ as in the lemma). Therefore, one may complete the proof by the same way as done in (3.15) (for the existence of such $h$, see also (3.15)).

B. We assume that $c(\operatorname{Fix}(h))=0$ and $c\left(\operatorname{Fix}\left(h^{2}\right)\right)=1$. $L$ may be given as $D^{2} \times S^{1} U_{f} S^{1} \times D^{2}$ where $D^{2} \times S^{1}$ is an $h$-invariant regular neighborhood of Fix $\left(h^{2}\right)$. Let $\left(\begin{array}{ll}a & c \\ b & d\end{array}\right)$ be the matrix of $f$.

LEMMA 4.17. $b=2 s$ for some odd $s$, and therefore $a$ is odd.

Proof. Using Theorem 2.9, one can show that $b$ is even and $2 d-b r \equiv 0$ mod 4 for some odd $r$ (see the proofs of Lemmas 3.4 and 4.14). Therefore, $b \neq 0 \bmod 4$.

By proceeding as in the second part of A, we may assume that $L=$ $L(a, c, b, d)$ and $h$ is given by

$$
\begin{array}{ll}
h\left(\rho z_{1}, z_{2}\right)=\left(\rho i z_{1}, z_{2}\right) & \text { on } D^{2} \times S^{1} \text { and } \\
h\left(z_{1}, \rho z_{2}\right)=\left(i z_{1},-\rho z_{2}\right) & \text { on } S^{1} \times D^{2} .
\end{array}
$$

Note that $a$ is odd. Let us denote this $h$ by $h(a, c, b, d)$. It is checked that the same statements as in Lemmas 3.8 and 3.9 are also true in this case (use the same homeomorphisms $t$ as in the lemmas; for the proof of (2) of Lemma 3.9 , note that $m \equiv 0 \bmod 4$ since both $b$ and $b+m p$ are even but not divisible by 4). Therefore, one can prove the following theorem in the same way as in (3.10) (for the existence of such $h$, note that there exist some integers $r, s$ such that $p r-2 s q=1$ since $p$ is odd and $(p, q)=1$; here we may assume that $s$ odd, and use the method in (3.10)).

THEOREM 4.18. If $c(\operatorname{Fix}(h))=0$ and $c\left(\operatorname{Fix}\left(h^{2}\right)\right)=1$, then $p$ is odd. Up to weak equivalence, there exists exactly one such $h$ on $L=L(p, q)$ ( $p$ odd) if $L$ is symmetric, and there exist exactly two such $h$ if $L$ is nonsymmetric.

Rice [17] showed that every free $Z_{4}$-action on $S^{3}$ is conjugate to the orthogonal action. This, along with Theorem $A$ and the above theorem, shows that:

THEOREM 4.19. $S^{3}$ admits exactly three orientation-preserving $Z_{4}$-actions, up to conjugation.

C. We assume that $c(\operatorname{Fix}(h))=1$ and $c\left(\operatorname{Fix}\left(h^{2}\right)\right)=2$. Again $L$ may be given as $D^{2} \times S^{1} \cup_{f} S^{1} \times D^{2}$ where $D^{2} \times S^{1}$ is an $h$-invariant regular neighborhood of Fix $(h)$. Let $\left(\begin{array}{l}a \\ b\end{array}\right)$ c $)$ be the matrix of $f$. Using Theorem 2.9, it can be seen that $a$ is even and $2 b+a r \equiv 0 \bmod 4$ (see the proofs of Lemmas 3.4 
and 4.14). Therefore, we see that $a=2 s$ for some odd $s$. By proceeding in the same spirit as in the previous cases, we may assume that $L=L(a, c, b, d)$ and $h$ is given by

$$
\begin{array}{ll}
h\left(\rho z_{1}, z_{2}\right)=\left(\rho i z_{1}, z_{2}\right) & \text { on } D^{2} \times S^{1} \text { and } \\
h\left(z_{1}, \rho z_{2}\right)=\left(-z_{1}, \rho i z_{2}\right) & \text { on } S^{1} \times D^{2} .
\end{array}
$$

Note that $b$ is odd. We denote this $h$ by $h(a, c, b, d)$. It is checked that the same statements as in Lemmas 3.8 and 3.9 are also true in this case. Therefore, one may conclude the following result (see (3.10)).

THEOREM 4.20. If $c(\operatorname{Fix}(h))=1$ and $c\left(\operatorname{Fix}\left(h^{2}\right)\right)=2$, then $p=2 s$ for some odd s. Up to weak equivalence, there exists exactly one such $h$ on each $L=L(2 s, q)(s$ odd) if $L$ is symmetric, and there exist exactly two if $L$ is nonsymmetric.

It follows from results of this section that there exists exactly one orientation-preserving nonfree $Z_{4}$-action on $P^{3}$, up to conjugation (see Theorem 4.20). Now suppose that $Z_{4}$ acts freely on $P^{3}$. Let $M=P^{3} / Z_{4}$. Then we see that $\pi_{1}(M)$ is an abelian group of order 8 . Therefore, it follows from a result of Epstein [3] that $\pi_{1}(M)=Z_{8}$. We may assume that $\pi_{1}(M)$ acts freely on $S^{3}$. Therefore, we have $M \approx L(8, q)$ for some $q$ (see Ritter [18]). Thus the possible orbit types are $L(8,1)$ and $L(8,3)$. Since there exist free $Z_{4}$-actions whose orbit spaces are $L(8,1)$ and $L(8,3)$, we have the following.

THEOREM 4.21. $P^{3}$ admits exactly three orientation-preserving $Z_{4}$-actions, up to conjugation.

\section{REFERENCES}

1. G. E. Bredon, Cohomological aspects of transformation groups, Proc. Conf. on Transformation Groups, Springer-Verlag, New York, 1968, pp. 245-280. MR 39 \#6303. 726.

2. S. Eilenberg, On the problems of topology, Ann. of Math. (2) 50 (1949), 247-260. MR 10,

3. D. B. A. Epstein, Finite presentations of groups and 3-manifolds, Quart. J. Math. Oxford Ser. (2) 12 (1961), 205-212. MR 26 \# 1867.

4. S. T. Hu, Homotopy theory, Academic Press, New York, 1959, p. 287. MR 21 \#5186.

5. P. K. Kim, PL involutions on lens spaces and other 3-manifolds, Proc. Amer. Math. Soc. 44 (1974), 467-473. MR 51 \#11558.

6. P. K. Kim and J. L. Tollefson, Splitting the PL involutions on nonprime 3-manifolds, Michigan Math. J. (to appear).

7. K. W. Kwun, Scarcity of orientation-reversing PL involutions of lens spaces, Michigan Math. J. 17 (1970), 355-358. MR 43 \#5535.

8. 73-77. MR 46 \# 9963.

9. G. R. Livesay, Fixed point free involutions on the 3-sphere, Ann. of Math. (2) 72 (1960), 603-611. MR 22 \#7131.

10. Involutions with two fixed points on the 3-sphere, Ann. of Math. (2) 78 (1963), 582-593. MR 27 \# 5257. 
11. W. Mangler, Die Klassen von Topologischen Abbildungen einer geschlossenen Fläche auf sich, Math. Z. 44 (1939), 541-554.

12. E. E. Moise, Affine structures in 3-manifolds. V: The triangulation theorem and Hauptvermutung, Ann. of Math. (2) 56 (1952), 96-114. MR 14, 72.

13. __ Periodic homeomorphisms of the 3-sphere, Illinois J. Math. 6 (1962), 206-225. MR 27 \# 755.

14. P. Olum, Mappings of manifolds and the notion of degree, Ann. of Math. (2) 58 (1953), 458-480. MR 15, 338.

15. C. D. Papakyriakopoulos, On Dehn's lemma and the asphericity of knots, Ann. of Math. (2) 66 (1957), 1-26. MR 19, 761.

16. K. Riedemeister, Homotopieringe und Linsenräume, Abh. Math. Sem. Univ. Hamburg 11 (1935), 102-109.

17. P. M. Rice, Free actions of $Z_{4}$ on $S^{3}$, Duke Math. J. 36 (1969), 749-751. MR 40 \#2064.

18. G. X. Ritter, Free actions of $Z_{8}$ on $S^{3}$, Trans. Amer. Math. Soc. 181 (1973), 195-212.

19. P. A. Smith, Periodic transformations of 3-manifolds, Illinois J. Math. 9 (1965), 343-348. MR 30 \#5311.

20. J. Stallings, On fibering certain 3-manifolds, Topology of 3-Manifolds and Related Topics, Prentice-Hall, Englewood Cliffs, N. J., 1962, pp. 95-100. MR 28 \# 1600.

21. J. L. Tollefson, Involutions on $S^{1} \times S^{2}$ and other 3-manifolds, Trans. Amer. Math. Soc. 183 (1973), 138-152. MR 48 \# 5081.

22. F. Waldhausen, On irreducible 3-manifolds which are sufficiently large, Ann. of Math. (2) 87 (1968), 56-88. MR 36 \#7146.

23. _ـ Über Inoolutionen der 3-sphäre, Topology 8 (1969), 81-91. MR 38 \#5209.

Department of Mathematics, Michigan State University, East lansing, Michigan 48824

Department of Mathematics, University of Rhode Island, Kingston, Rhode Island 02881

Current address: Department of Mathematics, University of Kansas, Lawrence, Kansas 66045 